\title{
Capital Asset Pricing under Ambiguity
}

\author{
Yehuda Izhakian*†
}

February 17, 2012

\begin{abstract}
This paper generalizes the mean-variance preferences to mean-variance-ambiguity preferences by relaxing the standard assumption that probabilities are known and assuming that probabilities are themselves random. It introduces a new measure of uncertainty, one that consolidates risk and ambiguity, which is employed for extending the CAPM from risk to uncertainty by incorporating ambiguity. This model makes the distinction between systematic ambiguity and idiosyncratic ambiguity and proves that the ambiguity premium is proportional to the systematic ambiguity. The merit of this model is twofold: first, it can be tested empirically; second, it can serve for measuring the performance of portfolios relative to their uncertainty.
\end{abstract}

Keywords and Phrases: Shadow Theory, Ambiguity, Ambiguity Measure, Uncertainty Measure, Ambiguity premium, Mean-variance, Mean-uncertainty, Capital Market Line (CML), Capital Asset Pricing Model (CAPM).

JEL Classification Numbers: D81, D83, G11, G12.

*Department of Economics, Stern School of Business, New York University, yud@stern.nyu.edu

${ }^{\dagger}$ We benefited from discussions with Menachem Abudy, Yakov Amihud, Pierpaolo Battigalli, Azi BenRephael, Simon Benninga, Menachem Brenner, Ilan Cooper, Alex Cukierman, Itamar Drechsler, Ignacio Esponda, Xavier Gabaix, Itzhak Gilboa, Eitan Goldman, William Greene, Sergiu Hart, Saggi Katz, Ilan Kremer, Yacov Oded, Thomas Sargent, David Schmeidler, Paul Wachtel and Avi Wohl. 


\section{Introduction}

The assumption underlying modern portfolio theory is that the probabilities of returns are known, such that there is a unique mean-variance space on which preferences are imposed and the capital asset pricing model (CAPM) is established. In reality probabilities are usually unknown and an additional premium is required to induce investors to bear ambiguity (Knightian uncertainty). ${ }^{1}$ What is the nature of this premium? Is it proportional to the entire, systematic and idiosyncratic, ambiguity? Or, is it proportional only to the systematic ambiguity? Can systematic ambiguity and idiosyncratic ambiguity be differentiated? The current paper is motivated by these questions.

This paper contributes to the existing literature in four ways. First, it introduces an objective empirically applicable measure of uncertainty that consolidates risk and ambiguity. Second, it generalizes the mean-variance space (Markowitz $(1952,1959))$ to a mean-uncertainty space, i.e., a mean-standard-deviation-ambiguity space, where uncertainty is considered to be the aggregation of risk and ambiguity. It establishes the efficient frontier and the capital market line (CML) and characterizes the mean-uncertainty preferences in this space. Third, the paper generalizes the CAPM to incorporate ambiguity while making the distinction between systematic ambiguity and idiosyncratic ambiguity. It proves that investors are rewarded for systematic uncertainty, but not for idiosyncratic uncertainty. Fourth, the paper introduces new performance measures by extending the Treynor and Sharpe ratios from risk to uncertainty.

Izhakian (2011), which introduces a new model of ambiguity, called shadow probability theory (henceforth shadow theory) and studies how it affects decision makers' choices, ${ }^{2}$ provides the theoretical underpinning of the current paper on the implication of ambiguity for capital asset pricing. The measure for the degree of ambiguity derived in Izhakian (2011) is a center piece of the theoretical model established in this paper. Shadow theory assumes that not only are the returns on assets random but the probabilities of these returns are themselves also random. The main idea of this theory is that, just as we measure the degree of risk by the variance of outcomes, so too can the degree of ambiguity be measured by the variance of the probability of loss (or gain). ${ }^{3}$ To demonstrate the merits of shadow theory for asset pricing, Izhakian (2011)

\footnotetext{
${ }^{1}$ Risk is defined as a situation in which the event to be realized is a-priori unknown, but the odds of all possible events are perfectly known. Ambiguity refers to conditions in which not only is the event to be realized a-priori unknown, but the odds of events are also either not uniquely assigned or are unknown.

${ }^{2}$ Shadow theory generalizes Schmeidler's (1989) Choquet expected utility and Tversky and Kahneman's (1992) cumulative prospect theory.

${ }^{3}$ Measuring risk by the variance of outcomes is admissible under some conditions; the same is true for measuring ambiguity by the variance of probabilities.
} 
proposes a well-defined ambiguity premium, which is completely separate from the conventional risk premium. This premium has been tested empirically by Brenner and Izhakian (2011), who show that ambiguity has a significant impact on stock market return. The present paper takes this line of research one step further by making the distinction between systematic ambiguity and idiosyncratic ambiguity and employing this distinction to refine the ambiguity premium.

The neoclassical finance literature dealing with capital asset pricing usually assumes away ambiguity and focuses on the risk-return relationship in the mean-standard-deviation space. The ability of this literature, including the widely used CAPM (Treynor (1961) and Sharpe (1964)), to record the full realistic picture of uncertainty has been challenged over the years. The current paper generalizes the mean-standard-deviation space to a mean-standard-deviationambiguity space and forms the relationships between risk, ambiguity and return. It relaxes the assumption that return are normally distributed with known parameters (mean and variance) and assumes that these parameters are themselves random. That is, ambiguity prevails and takes the form of random probabilities, dominated by second-order probabilities. As investors are assumed to be ambiguity averse, the reduction between first-order and second-order probabilities is relaxed. To combine investors' beliefs regrading outcomes and their probabilities, the paper introduces a new measure of uncertainty, which aggregates risk and ambiguity into a single consolidated measure. Given an expected return, in our model, optimal portfolios are those with the minimal degree of uncertainty.

This paper introduces a novel capital asset pricing model, called the shadow capital asset pricing model (SCAPM), which proves that the total uncertainty of an asset is not the relevant determinant of its price but only the systematic component. The SCAPM makes the distinction between systematic ambiguity, dominated by economy-wide shocks, and idiosyncratic ambiguity, dominated by firm-specific shocks. ${ }^{4}$ Formally, the ambiguity premium is extracted by introducing a beta ambiguity, in addition to the conventional beta risk. The SCAPM asserts that the expected return on an asset depends on the correlation of its probability of loss with the probability of loss on the market portfolio and not on the ambiguity associated with its own probability fluctuations. A high degree of ambiguity is not necessarily accompanied by a higher expected return, which is a function of the systematic component of ambiguity, measured by the beta ambiguity with respect to the market portfolio. Since risk and ambiguity are usually negatively related (see Izhakain (2012)), in SCAPM the systematic risk and the systematic

\footnotetext{
${ }^{4}$ The CAPM makes the distinction between systematic risk, for which investors are rewarded via a higher rate of return, and idiosyncratic risk, which is not accompanied by an additional reward.
} 
ambiguity are the optimal and not necessarily the minimal for a given expected return. Their aggregation to systematic uncertainty, however, is the minimal possible degree of uncertainty for a given level of expected return. A special case of the SCAPM is when probabilities are known, i.e., the degree of ambiguity is zero. In this case the beta ambiguity equals zero, the model collapses to the classical CAPM and the optimal portfolio has minimal risk for a given expected return.

Decomposing uncertainty into systematic and idiosyncratic components allows for extending the Sharpe ratio and the Treynor ratio, which are commonly used for evaluating portfolio performance, from risk to uncertainty. The Sharpe ratio evaluates the premium per unit of the entire, systematic and idiosyncratic, risk borne by an asset, while the Treynor ratio evaluates the premium per unit of systematic risk borne. Our extended performance measures evaluate the uncertainty premium per unit of total, systematic and idiosyncratic, uncertainty borne and the premium per unit of systematic uncertainty borne.

The theoretical implications of ambiguity for asset pricing have been studied mainly in the context of the equity premium. Chen and Epstein (2002), Cao et al. (2005), Nau (2006), Izhakian and Benninga (2011) and Ui (2011), for example, focus on decomposing the equity premium into two components: risk premium and ambiguity premium. Dow and Werlang (1992), Cao et al. (2005) and Easley and O'Hara (2009) attribute limited market participation to ambiguity aversion and study its impact on the equity premium. Uppal and Wang (2003), Maenhout (2004), Taboga (2005) and Gollier (2011), for example, study issues of optimal portfolio selection under ambiguity. Leippold et al. (2008), Gagliardini et al. (2009) and Ju and Miao (2011), for example, tie ambiguity to puzzling financial phenomena - such as the equity premium puzzle, the risk-free rate puzzle and the excess volatility puzzle. ${ }^{5}$ Unlike these papers, which consider the ambiguity premium of an asset independently of ambiguity of other assets in the market, the current paper studies the nature of asset ambiguity relative to market ambiguity.

Several extension of the mean-variance approach to ambiguity have been suggested by the literature. Pflug and Wozabal (2007) add ambiguity to the mean-variance preferences by applying the max-min approach of Gilboa and Schmeidler (1989) to a confidence set of probability distributions. Boyle et al. (2011) assume a mean-variance space with known variances and unknown mean. The SCAPM is broader: it assumes an unknown mean and an unknown variance. An unknown variance plays an important role in ambiguity, especially where

\footnotetext{
${ }^{5}$ For a recent survey on ambiguity in asset pricing and portfolio choice see Guidolin and Rinaldi (2010).
} 
an asset portfolio is concerned. The importance of random variance is stressed by Bollerslev et al. (1988), who show that conditional covariances are quite variable over time and are a significant determinant of time-varying risk premia.

A study that is related to this paper, Maccheroni et al. (2011), defines the ambiguity premium, referred to as alpha, by the residual between expected return and the risk premium reward for systematic risk. In an earlier, Epstein and Schneider (2008) show that the ambiguity premium depends on the idiosyncratic risk in fundamentals, which is practically equivalent to the alpha proposed by Maccheroni et al. (2011). Even though the SCAPM is based on a different decision-making model, it adds to these papers by providing a closed-form solution for deriving the alpha and showing that it can be explained as the reward for systematic ambiguity. ${ }^{6}$

Chen and Epstein (2002) generalize the consumption CAPM by building dynamic recursive multiple prior max-min preferences. Maccheroni, et al. (2009) use variational preferences to derive a version of the CAPM that under monotone mean-variance preferences can be generalized to incorporate ambiguity. ${ }^{7}$ In these models an asset's beta is derived by the covariance between its return and the pricing kernel, which makes no distinction between risk and ambiguity. Unlike these models, the SCAPM achieves a complete separation between risk and ambiguity and attains a well-defined beta ambiguity entirely separated from the beta risk.

Merton (1973) introduces a dynamic version of the CAPM and shows that the expected returns on risky assets may differ from the risk-free rate even when these assets do not have systematic risk. He attributes this difference to shifts in the investment opportunity set correlated with a zero-beta portfolio. SCAPM suggests an alternative explanation; it attributes the difference between expected returns on assets with zero systematic risk and the risk-free rate to the presence of ambiguity. In particular, it suggests that this additional expected return is proportional to the beta ambiguity. ${ }^{8}$

The theoretical model introduced in this paper paves the way for further, especially empirical, research into the risk-ambiguity-return relationship. The beta risk and the beta ambiguity are both empirically testable.

The rest of the paper is organized as follows. For completeness, Section 2 reviews the main principles of shadow theory. Section 3 extends the classical mean-standard-deviation space

\footnotetext{
${ }^{6}$ Maccheroni et al.'s (2011) model is based on Klibanoff et al.'s (2005) smooth model of ambiguity and its recursive form (Klibanoff et al.(2009)). Epstein and Schneider's (2008) model is based on Gilboa and Schmeidler's (1989) max-min model.

${ }^{7}$ The Giloba and Schmeidler (1989) and Hansen and Sargent (2001) models are special cases of variational preferences.

${ }^{8}$ The CAPM has been adjusted to other sources of risk. For example, Acharya and Pedersen (2005) introduce a liquidity-adjusted CAPM consisting of beta risk and beta liquidity.
} 
to mean-standard-deviation-ambiguity and forms preferences. Section 4 builds the efficient frontier and the CML in the mean-uncertainty settings. Section 5 generalizes the CAPM to incorporate ambiguity and discusses the implications of various components of ambiguity for capital asset pricing. Section 6 discusses the security market line (SML) and performance measures. Section 7 concludes.

\section{The model of ambiguity}

Izhakian (2011) introduce a novel ambiguity measure derived from a new model of decision making under ambiguity, called shadow probability theory, which provides the theoretical underpinning of this paper. We first review its main principles and its basic implementation for asset pricing.

\subsection{Shadow theory}

Shadow theory generalizes Schmeidler's (1989) Choquet expected utility by adding referencedependent beliefs. Like Tversky and Kahneman's (1992) cumulative prospect theory, it assumes that investors have a reference point relative to which outcomes are classified as a loss or as a gain. ${ }^{9}$ Consequences lower than the reference point are considered as a loss and consequences higher than the reference point are considered as a gain. The cumulative probability of loss events plays an important role in measuring the degree of ambiguity.

Shadow theory assumes two tiers of uncertainty, one with respect to outcomes and the other with respect to the probabilities of these outcomes, each tier of uncertainty being modeled by a separate state space. This structure introduces a complete distinction of risk from ambiguity with regard to both beliefs and preferences. The degree of ambiguity and the investor's attitude toward it are then measured with respect to one space, while risk and the investor's attitude toward it apply to the second space. As a consequence of random probabilities and the nonlinear ways in which individuals may interpret probabilities, perceived probabilities are nonadditive. Ambiguity aversion results in a subadditive probability measure, while ambiguity seeking results in a superadditive measure. ${ }^{10}$

The main idea of shadow theory is that the probabilities of outcomes are random; thus, just

\footnotetext{
${ }^{9}$ Cumulative prospect theory generalizes the original prospect theory of Kahneman and Tversky (1979) from risk to uncertainty. It modifies the probability weightings to allow a state space with an infinite support and to solve issues related to stochastic dominance.

${ }^{10}$ Nonadditivity means that probabilities do not necessarily add up to unity.
} 
as the degree of risk can be measured by the variance of outcomes, the degree of ambiguity can be measured by the variance of probabilities. However, where the variance of probabilities is concerned, the question is: to the probability of which event does the variance apply? Given a classification of outcomes as a loss or as a gain, Izhakian (2011) proves that the degree of ambiguity can be measured as four times the variance of the cumulative probability of loss, which is equal to four times the variance of the cumulative probability of gain.

In asset pricing ambiguity implies a premium, called the ambiguity premium, in addition to the conventional risk premium. The risk premium is the premium that investors are willing to pay for replacing a risky bet by its expected outcome. The ambiguity premium is the premium that investors are willing to pay for replacing an ambiguous bet, i.e., a bet with unknown probabilities, by a risky bet, i.e., a bet with known probabilities, having an identical expected outcome. The uncertainty premium is the total premium that investors are willing to pay for replacing an ambiguous bet by its expected outcome, i.e., the accumulation of the risk premium and the ambiguity premium.

An investor is ambiguity averse if she prefers the expectations of the random probability of an outcome over the random probability itself. These preferences concerning ambiguity are modeled by a continuous twice-differentiable function $\psi(\cdot)$, called the sake function. Ambiguity aversion takes the form of a concave sake function, while ambiguity loving takes the form of a convex sake function and ambiguity neutrality the form of a linear sake function. Preferences concerning risk are modeled by a continuous twice-differentiable utility function $\mathrm{U}(\cdot)$. As usual, risk aversion takes the form of a concave utility function, risk loving the form of a convex utility function and risk neutrality the form of a linear utility function. The Arrow-Pratt coefficient of absolute risk aversion is then defined by $-\frac{\mathrm{U}^{\prime \prime}(\cdot)}{\mathrm{U}^{\prime}(\cdot)}$. Similarly, the coefficient of absolute ambiguity aversion is defined by $-\frac{\psi^{\prime \prime}(\cdot)}{\psi^{\prime}(\cdot)}$.

Let $r \in \mathbb{R}$ be the random and ambiguous return on an asset. The uncertainty premium $\mathcal{K}$, derived by shadow theory, is formed by

$$
\mathcal{K} \approx \underbrace{-\frac{1}{2} \frac{\mathrm{U}^{\prime \prime}(\mathrm{E}[r])}{\mathrm{U}^{\prime}(\mathrm{E}[r])} \operatorname{Var}[r]-\frac{1}{8}\left[\frac{\psi^{\prime \prime}\left(\mathrm{E}\left[\mathrm{P}_{L}\right]\right)}{\psi^{\prime}\left(\mathrm{E}\left[\mathrm{P}_{L}\right]\right)}+\frac{\psi^{\prime \prime}\left(\mathrm{E}\left[\mathrm{P}_{G}\right]\right)}{\psi^{\prime}\left(\mathrm{E}\left[\mathrm{P}_{G}\right]\right)}\right] \mho^{2}[r]}_{\mathcal{R}},
$$

where $\mathcal{R}$ is the risk premium and $\mathcal{A}$ is the ambiguity premium. The parameters $\mathrm{P}_{L}$ and $\mathrm{P}_{G}$ are the random probabilities of loss and gain, respectively. Their expectations, $\mathrm{E}\left[\mathrm{P}_{L}\right]$ and $\mathrm{E}\left[\mathrm{P}_{G}\right]$, are taken with respect to the second-order probability distribution. That is,

$$
\mathrm{E}\left[\mathrm{P}_{L}\right]=\sum_{i} \chi_{i} \mathrm{P}_{i}\left(r<r_{k}\right) \quad \text { and } \quad \mathrm{E}\left[\mathrm{P}_{G}\right]=\sum_{i} \chi_{i} \mathrm{P}_{i}\left(r \geq r_{k}\right)
$$


where $r_{k}$ is the reference return which distinguishes losses from gains, and $\chi_{i}$ is the probability of the probability distribution $\mathrm{P}_{i}$.

The expected return, E $[r]$, and the variance of return, $\operatorname{Var}[r]$, are evaluated using expected probabilities, i.e., a double expectation of the random probability of return and the second-order probabilities. The component

$$
\mho^{2}[r]=4 \operatorname{Var}\left[\mathrm{P}_{L}\right]=4 \operatorname{Var}\left[\mathrm{P}_{G}\right]
$$

is Izhakian's measure of ambiguity, which is four times the variance of the probability of loss or four times the variance of the probability of gain, taken with respect to the second-order probability distribution $\chi$. It is important to note that $\mho^{2} \in[0,1]$ attains its minimal value, 0 , when all probabilities are known, and its maximal value, 1 , only in the extreme case of a binomial distribution with a random probability of each event that can take the probabilities 0 or 1 with equal likelihood.

\subsection{Illustration}

To illustrate the concept of ambiguity in the context of shadow theory, let us consider the following binomial example of an asset with two possible future returns: $d=-10 \%$ and $u=$ $20 \%$. Assume for the moment that the probabilities of $d$ and $u$ are known, say $\mathrm{P}(d)=\mathrm{P}(u)=$ 0.5. The average return is, thus, $5 \%$ and the standard deviation of return, which proxies for the degree of risk, is $15 \%$. Obviously, in this case, since probabilities are precisely known, ambiguity is not present and investors face only risk.

Assume now that the probabilities of $d$ and $u$ can be either $\mathrm{P}(d)=0.4$ and $\mathrm{P}(u)=0.6$ or alternatively $\mathrm{P}(d)=0.6$ and $\mathrm{P}(u)=0.4$, where these two alternative distributions are equally likely. This means that investors now face not only risk but also ambiguity, which can be measured using the variance of the probability of loss. Computing this variance yields $\operatorname{Var}\left[\mathrm{P}_{L}\right]=0.01$, which in turn implies a degree of ambiguity of $\mho=0.2$. Notice that the degree of risk has not changed since the variance is computed using the expected probabilities $\mathrm{E}\left[\mathrm{P}_{d}\right]=\mathrm{E}\left[\mathrm{P}_{u}\right]=0.5$.

If we consider, for example, investors of the constant relative risk aversion (CRRA) type and the constant absolute ambiguity aversion (CAAA) type, with the coefficient of risk aversion $\gamma=2$ and the coefficient of ambiguity aversion $\eta=2$, Equation (1) then becomes

$$
\mathcal{K} \approx \frac{1}{2} \gamma \operatorname{Var}[r]+\frac{1}{4} \eta \mho^{2}[r]=\operatorname{Var}[r]+\frac{1}{2} \mho^{2}[r]
$$


The uncertainty premium when probabilities are known consists only of the risk premium and satisfies $\mathcal{K}=\operatorname{Var}[r]=2.25 \%$. The price of this alternative reflects a total return of $7.25 \%$. In the second alternative, when probabilities are unknown, the uncertainty premium is $\mathcal{K}=\operatorname{Var}[r]+\frac{1}{2} \mho^{2}[r]=2.25 \%+2.0 \%=4.25 \%$, and the price reflects a total expected return of $9.25 \%$.

\section{The mean-standard-deviation-ambiguity space}

Underpinned by shadow theory, this section relaxes the standard assumption of modern portfolio theory (MPT) that probabilities of return are known and assumes that these probabilities are themselves random. It generalizes the mean-standard-deviation space to mean-standarddeviation-ambiguity and analyzes the tradeoff between expected return, risk and ambiguity.

Assuming mean-standard-deviation preferences, MPT introduced by Markowitz $(1952,1959)$ and Tobin (1958) asserts that a rational investor in an efficient markets selects a portfolio of assets that maximizes expected return for a given amount of risk, measured by the standard deviation of return. The main notion underlying MPT is the concept of diversification, which asserts that the collectively risk of a portfolio of non-perfectly positively correlated assets is lower than the risk of the individual assets. To allow preferences to be of the mean-standarddeviation type and risk to be measured by the standard deviation of return, MPT usually assumes that returns are normally distributed, so that probability distributions are completely characterized by a known mean and a known variance.

Returns on assets, in our model, are assumed to be normally distributed, but the parameters, governing the distribution, namely mean and variance, are assumed to be random. ${ }^{11}$ Formally, the normal probability distribution of an asset's return, $\mathrm{P}_{i}$, is governed by a random mean $\mu$ and a random standard deviation $\sigma$, designated $i=1, \ldots, m$. ${ }^{12}$ The reference point which distinguishes losses from gains, agreed upon by all investors, is the risk-free rate of return, denoted $r_{f}$. All assets are evaluated by their returns relative to $r_{f}$. Any return lower than $r_{f}$, even if it is positive, is considered as a loss and any return higher than $r_{f}$ is considered as a

\footnotetext{
${ }^{11}$ This assumption can be replaced by assuming that the utility function is either quadratic or of the constant absolute risk aversion type, for which preferences concern only the first two moments of the distribution. See, for example, Ljungqvist and Sargent (2004, 154-155). This assumption can also be replaced by assuming an elliptical distribution, which is characterized by the first two moments, mean and variance.

${ }^{12}$ Along this paper Greek letters stand for random variables and when the context is clear the index $i$ designating a possible realization is omitted.
} 
gain. The degree of ambiguity is then measured by

$$
\mho^{2}[r]=4 \operatorname{Var}\left[\int_{-\infty}^{r_{f}} \frac{1}{\sqrt{2 \pi \sigma^{2}}} e^{-\frac{(r-\mu)^{2}}{\sigma^{2}}} d r\right]=4 \operatorname{Var}\left[\Phi\left(r_{f} ; \mu, \sigma\right)\right],
$$

where $\Phi(\cdot)$ stands for the cumulative normal probability distribution.

To generalize the mean-variance space to mean-uncertainty, the following aggregation of the risk and the ambiguity measures to a consolidated uncertainty measure is proposed.

Model 3.1. The aggregated measure of risk and ambiguity, called the uncertainty measure, is defined by

$$
\nabla[r] \equiv \sqrt{\frac{\operatorname{Var}[r]}{1-\mho^{2}[r]}}
$$

Model 3.1 provides a unified measure of uncertainty in units of return. This measure forms a mapping from $[0,1]$, determined by the measure of ambiguity $\mho^{2}[r]$, to $[0, \infty)$. When no ambiguity is present $\mho^{2}[r]=0$ and the uncertainty measure collapses to the simple standard deviation, which proxies risk. In the second extreme case, when $\mho^{2}[r]=1$, the degree of uncertainty is infinite. An infinite degree of uncertainty is also attained when the standard deviation tends to infinity. It is important to note that $\nabla[\cdot]$ is an objective measure, which captures only beliefs, so that subjective preferences are not involved in measuring the degree of uncertainty. ${ }^{13}$

The mean-uncertainty space is built in two steps. First, the mean-standard-deviationambiguity space in $\mathbb{R}^{3}$ is established. Then, in a second step, using the uncertainty measure $\nabla[\cdot]$, triplets in $\mathbb{R}^{3}$ are projected to $\mathbb{R}^{2}$, defining a subspace, refereed to as the mean-uncertainty space. Preferences defined in the mean-standard-deviation-ambiguity space induce the preferences in the mean-uncertainty space.

The mean-standard-deviation-ambiguity space is a subset of $\mathbb{R}^{3}$ defined by the mean $\mathrm{E}[r]$, the standard deviation Std $[r]$, and the normalized ambiguity $\widehat{\mho}[r]$, which is formed as follows:

$$
\widehat{\mho}[r] \equiv \operatorname{Std}[r] \sqrt{\frac{\mho^{2}[r]}{1-\mho^{2}[r]}} .
$$

This normalization is applied for two reasons. The first is that since ambiguity $\mho$ is measured in units of probabilities, while $\mathrm{E}[r]$ and $\operatorname{Std}[r]$ are in units of return; Equation (7) normalizes $\mho$ to the units of return. Second, $\mho$ ranges between 0 and 1 , while $\mathrm{E}[r]$ and $\operatorname{Std}[r]$ range between 0 and $\infty$; Equation $(7)$ maps $\mho$ to $[0, \infty)$.

A portfolio $\mathbf{h}=\left(h_{1}, \ldots, h_{n}\right)$, consisting of $n$ assets with a proportion $h_{j} \in \mathbb{R}$ of asset $j$, is rep-

\footnotetext{
${ }^{13}$ Section 4 elaborates on the construction of $\nabla[\cdot]$.
} 
resented in the mean-standard-deviation-ambiguity space by a triplet $\left(\mathrm{E}\left[r_{\mathbf{h}}\right], \operatorname{Std}\left[r_{\mathbf{h}}\right], \widehat{\mho}\left[r_{\mathbf{h}}\right]\right) \in$ $\mathbb{R}^{3}$, where $r_{\mathbf{h}}=\sum_{j=1}^{n} h_{j} r_{j}$. Assuming for the moment that the risk-free asset in not available, the set of feasible portfolios can be defined by the set of parametric triplets $\mathbb{S}=\left\{\left(\mathrm{E}\left[r_{\mathbf{h}}\right], \operatorname{Std}\left[r_{\mathbf{h}}\right], \widehat{\mho}\left[r_{\mathbf{h}}\right]\right) \mid \sum_{j} h_{j}=1\right\} .{ }^{14}$ Each point in $\mathbb{S}$, defined by $\mathrm{E}\left[r_{\mathbf{h}}\right], \operatorname{Std}\left[r_{\mathbf{h}}\right]$ and $\widehat{\mho}\left[r_{\mathbf{h}}\right]$, designates an investment opportunity. Markets are incomplete: the set of feasible portfolios is less than the whole $\{\mathrm{E}, \mathrm{Std}, \widehat{\mho}\}$, i.e., $\mathbb{S} \subset \mathbb{R}^{3}$. To show this, the case of two perfectly correlated returns with different means has to be ruled out. This case implies that one could short one asset, long the other asset, and create an infinite expected return with no uncertainty. But, such a case is a violation of the law of one price, which must be satisfied since markets are in equilibrium. In other words, the law of one price implies that there is a bounded set of feasible portfolios in the mean-standard-deviation-ambiguity space. ${ }^{15}$

In a two-asset economy the set of feasible portfolios, $\mathbb{S}$, draws a curve in the mean-standarddeviation-ambiguity space; for three or more assets, conditional on the relationships between their probability moments, it draws a surface or a volume. For example, if no ambiguity is present, $\mathbb{S}$ draws a plane in the mean-standard-deviation-ambiguity space. $\mathbb{S}$ is not necessarily convex over the entire domain defined by the parameter $\mathbf{h}$, i.e., it can possibly be non-convex for a subdomain of $\mathbf{h}$. $\mathbb{S}$ is bounded by a hyperbola shape in the mean-standard-deviation section. In the mean-ambiguity section $\mathbb{S}$ is also bounded but not necessarily by a concave shape. The upper boundary of $\mathbb{S}$, refer to as the uncertain asset frontier, takes the shape of a curve or a surface in $\mathbb{R}^{3}$. A portfolio that lies on the uncertain asset frontier is denoted e.

Investors are assumed to be risk averse and ambiguity averse, and characterized by the utility function $\mathrm{U}(\cdot)$ and the sake function $\psi(\cdot)$, respectively, which are both monotonically increasing, concave and twice differentiable. Their decisions are considered in the context of a static model: investments are made in the first period, and the outcomes occur in the second period. Investors are assumed to maximize the expected utility of the end-of-period consumption. Since life ends at the second period, there is no difference between consumption and wealth: all end-of-period wealth is consumed. Investors can borrow or lend unlimited quantities at the risk-free rate of return, $r_{f}$, which is exogenous (see, for example, Sharpe (1964)). All available assets for trading are risky and ambiguous, except for the risk-free asset, which has a constant rate of return across all states of nature. All the assumptions of the CAPM are maintained, except for the assumption that the probabilities of return are known. ${ }^{16}$

\footnotetext{
${ }^{14}$ In this case, the zero return can be taken as the reference point.

${ }^{15}$ For a formal proof see Theorem 4.2 below.

${ }^{16}$ The CAPM assumes that markets are efficient in the sense that all information is available to all investors,
} 
Given the decision to save an amount $w$ of her welfare, an investor faces the decision regarding the composition of her optimal portfolio, i.e., the proportions of the risk-free asset and the uncertain assets. Given two portfolios with identical risk and ambiguity, a rational (risk and ambiguity averse) investor prefers the portfolio with the higher expected return; given two portfolios with identical expected return and risk, she prefers the portfolio with the lower degree of ambiguity; given two portfolios with identical expected return and ambiguity, she prefers the portfolio with the lower degree of risk.

Higher expected return shifts the distribution of future consumption toward higher levels, implying a higher expected utility. A symmetric risk increment implies higher probabilities of extreme events, which in turn implies a lower expected utility, since investors are risk-averse. ${ }^{17}$ Particularly, the expected utility generated by a normally distributed portfolio is a declining function of the standard deviation of return. ${ }^{18}$ A second source of uncertainty is ambiguity, which also has a negative impact on expected utility. Probabilities shaped by ambiguity-averse investors are subadditive, which means that they are a decreasing function of the degree of ambiguity $\mho^{2}$ and aversion to it. ${ }^{19}$ Preferences toward risk and toward ambiguity define a set of portfolios over which the investor is indifferent. Each such set draws a hull in $\mathbb{R}^{3}$, referred to as the indifference surface. An investor chooses from among all feasible portfolios the one placing him on the indifferent surface representing the highest level of utility.

Assuming that there are at least three ambiguous-risky assets, Figure 1 illustrates the set of feasible portfolios and the indifference surface in the mean-standard-deviation-ambiguity space in an economy without a risk-free asset. The upper concave shell represents the indifference surface describing the tradeoff, derived by preferences, between risk, ambiguity, and expected return. The higher the indifference surface the higher the level of expected utility. The lower, horizontal conic volume represents all feasible portfolios.

The tradeoff between the unexpected return of portfolio $\mathbf{h}, r_{\mathbf{h}}-\mathrm{E}\left[r_{\mathbf{h}}\right]$, and its degree of

including possible variances, possible covariances, possible mean rates of returns and all the other parameters. All investors have equal access to all securities in a market with no taxes and no commissions, and can short any asset and hold any fraction of any asset. They behave competitively and are faced with a perfect capital market in the sense that they can buy and sell as much as they want of any asset without affecting its price.

${ }^{17}$ Risk aversion implies that increasing the probabilities of high consumption levels and low consumption levels by the same magnitude has a negative impact on expected utility.

${ }^{18}$ For a detailed discussion about mean-variance preferences see, for example, Fama and Miller (1972 ).

${ }^{19}$ Subadditive means that probabilities add up to a number lower than 1. 


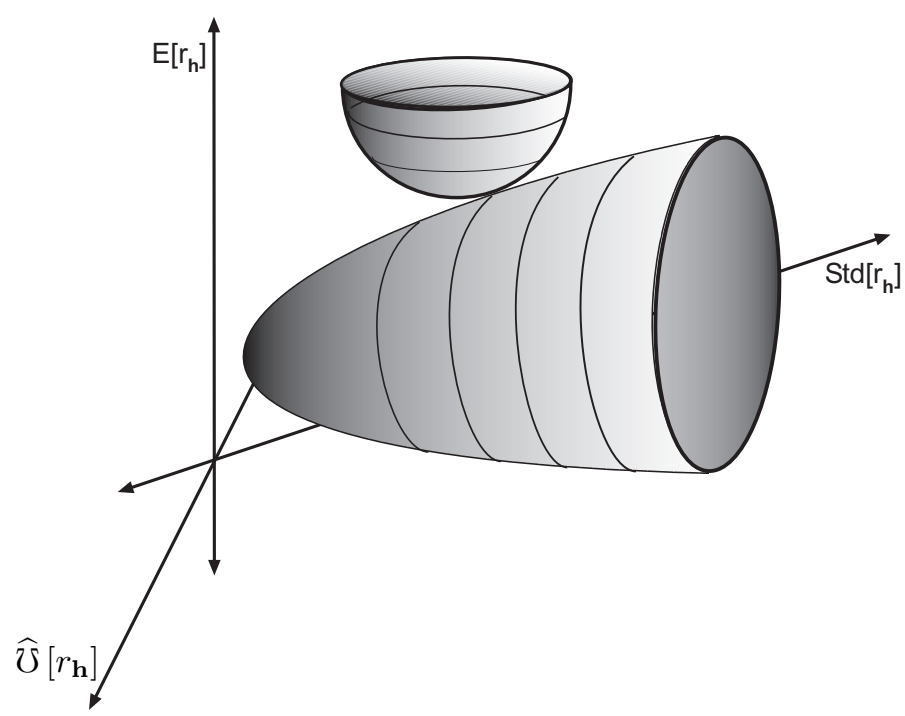

Figure 1: The feasible portfolios and the indifferent surface.

This figure illustrates the set of feasible portfolios in the mean-standard-deviation-ambiguity space, i.e., in $\mathbb{R}^{3}$, in an economy without a risk-free asset. The $\mathrm{x}$-axis describes the degree of risk, measured by the standard deviation. The y-axis describes the normalized degree of ambiguity. The z-axis describes the expected return. The upper concave shell is the indifference surface and the lower, horizontal conic volume represents the feasible portfolios.

uncertainty can be formulated by ${ }^{20}$

$$
R_{\mathbf{h}}=\frac{r_{\mathbf{h}}-\mathrm{E}\left[r_{\mathbf{h}}\right]}{\sqrt{\frac{\operatorname{Var}\left[r_{\mathbf{h}}\right]}{1-\mho^{2}\left[r_{\mathbf{h}}\right]}}}
$$

where the normalized unexpected return $R_{\mathrm{h}}$ is the random net unexpected return per unit of uncertainty borne. Notice that $R_{\mathbf{h}}$ is normally distributed, since $r_{\mathbf{h}}$ is normally distributed.

Assuming a two-period economy, the future consumption $c$ conditional on saving $w$ is determined by the one-period portfolio's return $r_{\mathbf{h}}$, defined by the terminal wealth $c=w\left(1+r_{\mathbf{h}}\right)$. Using Equation (8) the future consumption can be formulated by

$$
c=w\left(1+\mathrm{E}\left[r_{\mathbf{h}}\right]+R_{\mathbf{h}} \operatorname{Std}\left[r_{\mathbf{h}}\right] \frac{1}{\sqrt{1-\mho^{2}\left[r_{\mathbf{h}}\right]}}\right) .
$$

Equation (8) makes it possible to express the expected utility in terms of $R_{\mathbf{h}}$, since the terminal wealth is determined by $R_{\mathbf{h}}$. The expected utility associated with the choice to save $w$ in portfolio $\mathbf{h}$ is then

$$
\mathrm{E}[\mathrm{U}(c)]=\sum_{i} \chi_{i} \int_{-\infty}^{\infty} \mathrm{U}\left(w\left(1+\mathrm{E}\left[r_{\mathbf{h}}\right]+R_{\mathbf{h}} \operatorname{Std}\left[r_{\mathbf{h}}\right] \frac{1}{\sqrt{1-\mho^{2}\left[r_{\mathbf{h}}\right]}}\right)\right) \phi\left(R_{\mathbf{h}} ; \mu_{i, \mathbf{h}}, \sigma_{i, \mathbf{h}}\right) d R_{\mathbf{h}},
$$

where $\mu_{\mathbf{h}}$ and $\sigma_{\mathbf{h}}$ are the random mean and variance of the normalized return $R_{\mathbf{h}}$, respectively, and $\phi\left(R_{\mathbf{h}} ; \mu_{\mathbf{h}}, \sigma_{\mathbf{h}}\right)$ stands for the normal probability density function of $R_{\mathbf{h}}$ conditional on $\mu_{\mathbf{h}}$ and $\sigma_{\mathbf{h}}$. Since expected utility depends entirely on $w, \mathrm{E}\left[r_{\mathbf{h}}\right], \operatorname{Var}\left[r_{\mathbf{h}}\right]$ and $\mho^{2}\left[r_{\mathbf{h}}\right]$, it can be

\footnotetext{
${ }^{20}$ Fama and Miller (1972) apply the same idea to the tradeoff between return and standard deviation.
} 
written as

$$
\mathrm{E}\left[\mathrm{V}\left(w, \mathrm{E}\left[r_{\mathbf{h}}\right], \operatorname{Std}\left[r_{\mathbf{h}}\right], \mho\left[r_{\mathbf{h}}\right]\right)\right]
$$

One can verify that the marginal expected utility with respect to $\mathrm{E}\left[r_{\mathbf{h}}\right]$ is positive:

$$
\frac{\partial \mathrm{E}[\mathrm{V}(\cdot)]}{\partial \mathrm{E}\left[r_{\mathbf{h}}\right]}=w \sum_{i} \chi_{i} \int_{-\infty}^{\infty} \frac{\partial \mathrm{U}(c)}{\partial c} \phi\left(R_{\mathbf{h}} ; \mu_{i, \mathbf{h}}, \sigma_{i, \mathbf{h}}\right) d R_{\mathbf{h}}>0 .
$$

That is, while all other parameters remain unchanged, expected utility is an increasing function of expected return. The marginal expected utility with respect to $\operatorname{Std}\left[r_{\mathbf{h}}\right]$ is negative:

$$
\frac{\partial \mathrm{E}[\mathrm{V}(\cdot)]}{\partial \mathrm{Std}\left[r_{\mathbf{h}}\right]}=w \frac{1}{\sqrt{1-\mho^{2}\left[r_{\mathbf{h}}\right]}} \sum_{i} \chi_{i} \int_{-\infty}^{\infty} \frac{\partial \mathrm{U}(c)}{\partial c} \phi\left(R_{\mathbf{h}} ; \mu_{i, \mathbf{h}}, \sigma_{i, \mathbf{h}}\right) R_{\mathbf{h}} d R_{\mathbf{h}}<0 .
$$

To see this, notice that $\mu_{\mathbf{h}}$ is relatively close to 0 , and, therefore, $\mathrm{E}\left[\mu_{\mathbf{h}}\right]=\sum_{i} \chi_{i} \mu_{\mathbf{h}} \approx 0$. Because the marginal utility is a decreasing function of consumption (a concave utility function) and $R_{\mathbf{h}}$ is symmetrically distributed around $\mu_{\mathbf{h}}$, the value obtained is negative. From the same considerations, the marginal expected utility with respect to $\mho\left[r_{\mathbf{h}}\right]$ is also negative:

$$
\frac{\partial \mathrm{E}[\mathrm{V}(\cdot)]}{\partial \mho\left[r_{\mathbf{h}}\right]}=w \operatorname{Std}\left[r_{\mathbf{h}}\right] \frac{\mho\left[r_{\mathbf{h}}\right]}{\left(1-\mho^{2}\left[r_{\mathbf{h}}\right]\right)^{\frac{3}{2}}} \sum_{i} \chi_{i} \int_{-\infty}^{\infty} \frac{\partial \mathrm{U}(c)}{\partial c} \phi\left(R_{\mathbf{h}} ; \mu_{i, \mathbf{h}}, \sigma_{i, \mathbf{h}}\right) R_{\mathbf{h}} d R_{\mathbf{h}}<0 .
$$

The conclusion that emerges from this analysis, which proves that risk and ambiguity have a negative impact on expected utility while expected return has a positive impact, is that expected return can be considered as compensation for bearing risk and ambiguity. This understanding allows for a defining of the mean-standard-deviation-ambiguity preferences, which in turn enables the definition of efficient portfolios. A portfolio $\mathbf{h}$, characterized by the mean $\mathrm{E}\left[r_{\mathbf{h}}\right]$, the standard deviation $\operatorname{Std}\left[r_{\mathbf{h}}\right]$, and the ambiguity $\mho\left[r_{\mathbf{h}}\right]$, is efficient if there is no other portfolio with the same standard deviation and the same ambiguity that has a higher expected return, $\mathrm{E}\left[r_{\mathbf{h}}\right]$. The set of efficient portfolios establishes the efficient frontier, which takes the shape of a surface in $\mathbb{R}^{3}$. Technically, in an economy without a risk-free asset the efficient frontier is the upper boundary of the set of feasible portfolios - the uncertain asset frontier - which can be constructed by employing numerical methods.

Including a risk-free asset draws infinitely many lines stretching from the point $\left(r_{f}, 0,0\right)$ and tangent to the uncertain asset frontier. Each line tangents the uncertain asset frontier at a different point $\left(\mathrm{E}\left[r_{\mathbf{e}}\right], \operatorname{Std}\left[r_{\mathbf{e}}\right], \widehat{\mho}\left[r_{\mathbf{e}}\right]\right)$ and satisfies

$$
\left(\mathrm{E}\left[h r_{f}+(1-h) r_{\mathbf{e}}\right], \operatorname{Std}\left[h r_{f}+(1-h) r_{\mathbf{e}}\right], \widehat{\mho}\left[h r_{f}+(1-h) r_{\mathbf{e}}\right]\right)
$$

where $\mathrm{E}\left[r_{\mathbf{e}}\right]$ is the expected return on portfolio e lying on the uncertain asset frontier. These tangent lines stretching from $r_{f}$ are linear in $h$, as the following proposition proves. 
Proposition 3.2. Assuming that the reference point is $r_{f}$ and that the efficient portfolio $e$ is normally distributed then the line drawn by the parametric triplet

$$
\left(\mathrm{E}\left[h r_{f}+(1-h) r_{e}\right], \operatorname{Std}\left[h r_{f}+(1-h) r_{e}\right], \widehat{\mho}\left[h r_{f}+(1-h) r_{e}\right]\right),
$$

is linear in $h$.

Proposition 3.2 proves that when a risk-free asset exists any efficient portfolio lies on a straight line stretching from $r_{f}$ and is tangent to the uncertain asset frontier. In such an economy the efficient frontier is drawn by the collection of these lines. Expected utility is an increasing function of $\mathrm{E}\left[r_{\mathbf{h}}\right]$ and a decreasing function of $\operatorname{Std}\left[r_{\mathbf{h}}\right]$ and $\mho\left[r_{\mathbf{h}}\right]$. Hence, expected utility maximization implies that any optimal portfolio must be a on the efficient frontier. The particular optimal portfolio is selected by each investor individually according to her preferences concerning risk and ambiguity.

Figure 2 illustrates the uncertain asset frontier and the efficient frontier in an economy in which a risk-free asset exists. The straight dashed lines originating from the risk-free rate point $r_{f}$ and tangent to the curved surface describe the set of efficient portfolios, i.e., the efficient frontier. Corresponding to her preferences concerning risk and ambiguity, a rational investor selects one of the portfolios in this set, which is determined by the tangency point of the indifference surface and the efficient frontier.

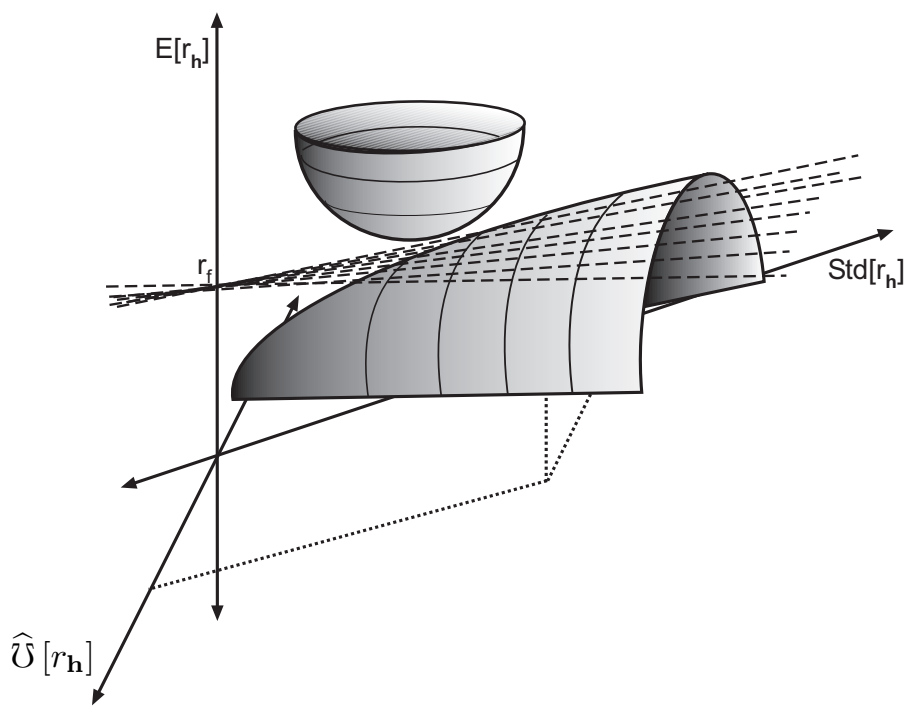

Figure 2: The uncertain asset frontier and the efficient frontier

This figure illustrates the uncertain asset frontier and the efficient frontier in the mean-standarddeviation-ambiguity space, i.e., in $\mathbb{R}^{3}$, when a risk-free asset exists. The $\mathrm{x}$-axis describes the degree of risk, measured by the standard deviation. The y-axis describes the normalized degree of ambiguity. The z-axis describes the expected return. The upper concave shell is the indifference surface and the lower, horizontal shaded concave surface is the uncertain asset frontier. The straight lines drawn from $r_{f}$ and tangent to the lower, horizontal concave surface describe the set of efficient portfolios. 


\section{The capital market line}

To construct the capital market line (CML) the mean-standard-deviation-ambiguity space is projected to the mean-uncertainty space in $\mathbb{R}^{2}$. For this purpose, the following assumption is made.

Assumption 4.1. All investors aggregate risk and ambiguity to uncertainty by Model 3.1 and have preferences concerning this uncertainty.

The consequence of this assumption is that all investors project the set of feasible portfolios, the uncertain asset frontier, and the efficient frontier in the same way, which implies that these three sets are unique in the mean-uncertainty space. It is important to note that Assumption 4.1 centers around beliefs and not around preferences. It asserts that all investors employ the same methodology to consolidate their beliefs regarding the degree of uncertainty based on their common beliefs regarding risk and ambiguity. As a result all investors see the same tradeoff between risk and ambiguity. Given their beliefs about the degree of uncertainty, each of the investors selects a portfolio depending upon their personal preferences concerning uncertainty. It is assumed that all investors solve the same optimization problem to maximize expected return conditional on the degree of uncertainty. Since all of them have the same investment opportunities to choose from, the same information, and the same decision procedure, every selected portfolio lies on the same efficient frontier.

The projection of the mean-standard-deviation-ambiguity space to the mean-uncertainty space is obtained by the mapping $(\mathrm{E}[r], \operatorname{Std}[r], \widehat{\mho}[r]) \mapsto(\mathrm{E}[r], \nabla[r])$, where $\nabla[r]$ stands for the degree of uncertainly. This mapping, defined by the Euclidean norm

$$
|(\operatorname{Std}[r], \widehat{\mho}[r])|=\sqrt{\operatorname{Var}[r]+\widehat{\mho}^{2}(r)},
$$

can be written (by substituting for $\widehat{\mho}$ ) as

$$
|(\operatorname{Std}[r], \widehat{\mho}[r])|=\sqrt{\frac{\operatorname{Var}[r]}{1-\mho^{2}[r]}}=\nabla[r],
$$

which is the uncertainty measure proposed by Model 3.1. Equation (11) maps every point $(\mathrm{E}, \mathrm{Std}, \widehat{\mho}) \in \mathbb{R}^{3}$ in the mean-standard-deviation-ambiguity space to a point $\left(\mathrm{E}, \frac{\mathrm{Std}}{\sqrt{1-\mho^{2}}}\right) \in \mathbb{R}^{2}$ in the mean-uncertainty space. Particulary, the uncertain asset frontier is mapped to a unique curve in the mean-uncertainty space; the efficient frontier is mapped to a unique line in the mean-uncertainty space called the capital market line (CML); and each indifference surface is mapped to a single curve in the mean-uncertainty space.

Assume for the moment a single uncertain asset, denoted $j$, and a risk-free asset. Figure 3 
shows the opportunity set available to the investors in the mean-uncertainty space. The slope of the opportunity line is given by

$$
\frac{d \mathrm{E}[r]}{d \nabla[r]}=\frac{\mathrm{E}\left[r_{j}\right]-r_{f}}{\nabla\left[r_{j}\right]},
$$

and its intercept is the risk-free rate. That is, the intercept is the portfolio consisting of only the risk-free asset, which is the only portfolio associated with a zero degree of uncertainty. The dashed line depicts the opportunities that are only possible if short sales are allowed.

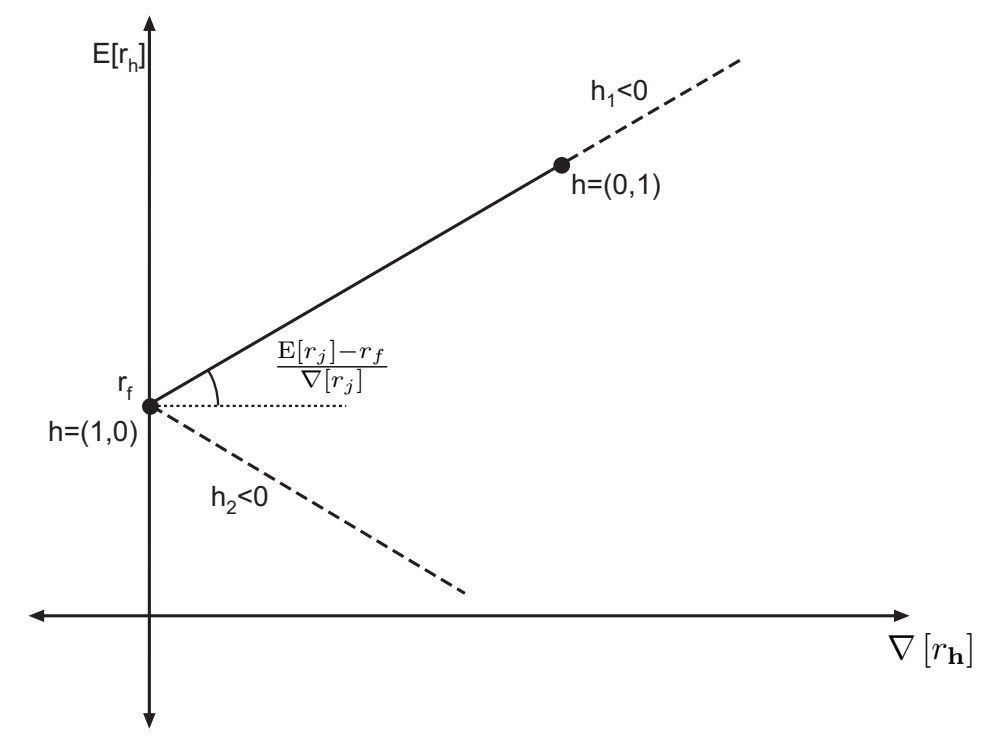

Figure 3: The opportunity set of one uncertain asset and a risk-free asset

This figure describes the opportunity set in the mean-uncertainty space, when there is only one uncertain asset and a risk-free asset. The $\mathrm{x}$-axis describes the degree of uncertainty, measured by $\nabla$, and the y-axis describes the expected return. $\mathbf{h}=(1,0)$ and $\mathbf{h}=(0,1)$ represent a portfolio consisting of only the risk-free asset and a portfolio consisting of only the uncertain asset, respectively. Portfolio shares range from zero to one along the solid portion of the opportunity set. Short sales of either asset extend the opportunity set along the dashed line.

As market are incomplete, the set of feasible portfolios in the mean-uncertainty space is a subset of $\mathbb{R}^{2}$. The uncertain asset frontier in the mean-uncertainty space takes the form of a curve which defines the minimal degree of uncertainty, $\nabla$, for every level of expected return. The degree of uncertainty and the expected return are always nonnegative so that this frontier lies in the first quadrant. The frontier exists since the law of one price is satisfied in equilibrium, such that there are no two perfectly correlated assets with different expected return. Eliminating purely redundant assets from consideration, the next theorem proves this formally.

Theorem 4.2. If the expected variance-covariance matrix of return is nonsingular and the degree of ambiguity is not equal to 1 , then the uncertain asset frontier and the efficient frontier exist. 
Writing the uncertain asset frontier as a function of expected return, i.e., the minimal degree of uncertainty as a function of expected return (see Equation (27)), shows that in almost all cases the frontier is concave over the entire domain. Exceptions, in which the curve is non-concave over two relatively small subdomains, might occur in the extreme case where the correlation between every two assets is close to 1 . This can happen since ambiguity and variance are not independent. The uncertain asset frontier is bounded by a hyperbola defined by the risk of the feasible portfolios. The following theorem proves this claim.

Theorem 4.3. The uncertain asset frontier is bounded by a hyperbola defined by risk, such that for any level of expected return the degree of uncertainty is higher than the degree of risk.

The CML takes the form of a line in $\mathbb{R}^{2}$ originating from $r_{f}$ and tangents the uncertain asset frontier at the point $\mathbf{m}$, which is referred to as the market portfolio or the tangency portfolio. In equilibrium the expected return on the market portfolio is at least as high as the risk-free rate. The risk-free rate is lower than the return on the portfolio with the minimal possible uncertainty; otherwise, investors with a mean-uncertainty objective would try to short the uncertain assets, which cannot represent an equilibrium (see Cochrane (2001)). All portfolios lying on the CML are efficient in the sense that they attain the minimal degree of uncertainty for a given level of expected return. It is important to note that in the mean-uncertainty space an efficient portfolio is not necessarily a portfolio with minimal risk for a given level of expected return.

The market portfolio, $\mathbf{m}$, is unique. To see this, note that since the market has already reached an equilibrium, which is governed by supply and demand, the proportion of any asset in the market portfolio is given by its capital market value, i.e., the total worth of its shares divided by the capital value of the whole market. The total worth of an asset's shares is unique, which implies that the proportion of each asset in the portfolio is unique and, therefore, the market portfolio is unique. The market portfolio, marked by the tangency point of the CML and the uncertain asset frontier, can be solved numerically by equating the slope of the CML to the slope of the uncertain asset frontier, which can be extracted from Equation (27).

Ambiguity and risk are usually negatively related (see Izhakian (2012)). Therefore, for a given expected return the variance of the efficient portfolio is at least as high as the minimal possible variance. Particularly, the market portfolio, $\mathbf{m}$, is not necessarily a portfolio with the minimal variance for a given expected return, but rather a portfolio with the minimal uncertainty. Consider two economies with identical parameters, except that one is typified by ambiguity and the other is not. If the expected return on the market portfolio is the same for 
the two economies, the volatility of the market portfolio in the ambiguous economy is not lower than that of the market portfolio in the non-ambiguous economy.

The one-period return $r_{\mathbf{m}}$ on the market portfolio $\mathbf{m}$ is random and ambiguous; it is normally distributed, governed by the random mean $\mu_{\mathbf{m}}$ and the random variance $\sigma_{\mathbf{m}}^{2}$, with an expected return $\mathrm{E}\left[r_{\mathbf{m}}\right] \geq r_{f}$. Any efficient portfolio $\mathbf{h}$ in the mean-uncertainty space consists of a proportion $(1-h)$ of the market portfolio and a proportion $h$ of the risk-free asset. Thus, the CML can be defined by the parameterized straight line $(\mathrm{E}(h), \nabla(h))$, where $\mathrm{E}(h)=(1-h) \mathrm{E}\left[r_{\mathbf{m}}\right]+h r_{f}$ is the expected return and $\nabla(h)=(1-h) \nabla\left[r_{\mathbf{m}}\right]$ is the degree of uncertainty. Considering a portfolio $\mathbf{h}$ consisting of a proportion $(1-h)$ of $\mathbf{m}$ and the remainder $h$ allocated to asset $j$, if $h=0$, then $(\mathrm{E}(0), \nabla(0))=\left(\mathrm{E}\left[r_{\mathbf{m}}\right], \nabla\left[r_{\mathbf{m}}\right]\right)$ and if $h=1$, then $(\mathrm{E}(1), \nabla(1))=\left(\mathrm{E}\left[r_{j}\right], \nabla\left[r_{j}\right]\right)$. The curve drawn by $(\mathrm{E}(h), \nabla[h])$ touches the CML at the market point $\left(\mathrm{E}\left[r_{\mathbf{m}}\right], \nabla\left[r_{\mathbf{m}}\right]\right)$, but otherwise remains off the CML, though, of course, within the feasible set of portfolios where it also hits the point $\left(\mathrm{E}\left[r_{j}\right], \nabla\left[r_{j}\right]\right)$.

In the mean-uncertainty space a rational investor minimizes the degree of uncertainty for a given expected return, such that any portfolio $\mathbf{h}$ she chooses lies on the CML. That is, any optimal portfolio satisfies

$$
\frac{\mathrm{E}\left[r_{\mathbf{h}}\right]-r_{f}}{\sqrt{\frac{\operatorname{Var}\left[r_{\mathbf{h}}\right]}{1-\mho^{2}\left[r_{\mathbf{h}}\right]}}}=\frac{\mathrm{E}\left[r_{\mathbf{m}}\right]-r_{f}}{\sqrt{\frac{\operatorname{Var}\left[r_{\mathbf{m}}\right]}{1-\mho^{2}\left[r_{\mathbf{m}}\right]}}} .
$$

The expected excess return over the risk-free rate, formed by $\mathrm{E}\left[r_{\mathbf{h}}\right]-r_{f}$, defines the uncertainty premium associated with portfolio $\mathbf{h}$, which is the reward for its uncertainty $\nabla\left[r_{\mathbf{h}}\right]=\sqrt{\frac{\operatorname{Var}\left[r_{\mathbf{h}}\right]}{1-\mho\left[r_{\mathbf{h}}\right]}}$. The CML defines the relationship between the expected return and the degree of uncertainty of a portfolio. Using Equation (12), the CML can be written as

$$
\mathrm{E}\left[r_{\mathbf{h}}\right]=r_{f}+\left(\frac{\mathrm{E}\left[r_{\mathbf{m}}\right]-r_{f}}{\nabla\left[r_{\mathbf{m}}\right]}\right) \nabla\left[r_{\mathbf{h}}\right],
$$

which implies a linear relationship between portfolio h's expected return $\mathrm{E}\left[r_{\mathbf{h}}\right]$ and its degree of uncertainty $\nabla\left[r_{\mathbf{h}}\right]$. The slope of the CML, $\left(\frac{\mathrm{E}\left[r_{\mathbf{m}}\right]-r_{f}}{\nabla\left[r_{\mathbf{m}}\right]}\right)$, defines the compensation per unit of uncertainty borne in the market. This compensation is the same for each investor, no matter how uncertainty averse she is.

The CML is steeper if the economy is less uncertain, i.e., if the returns on the market portfolio or its probabilities are less volatile. The reason is that investors ask for a relatively high premium for bearing assets' uncertainty when the alternative - the market portfolio - is associated with a relatively low uncertainty. A portfolio with $\nabla\left[r_{\mathbf{h}}\right]=0$ corresponds to $h=1$, i.e., a portfolio consisting of only the risk-free asset, which implies that its expected return 
is the risk-free rate. On the other hand, $h=0$ corresponds to a portfolio consisting of only the market portfolio and, thus, its expected return satisfies $\mathrm{E}\left[r_{\mathbf{h}}\right]=\mathrm{E}\left[r_{\mathbf{m}}\right]$. All other efficient portfolios are obtained along the CML.

The indifference surface in the mean-standard-deviation-ambiguity space is also projected to the mean-uncertainty space using Equation (11). This projection is valid since expected utility is positively affected by expected return and negatively affected by uncertainty, which is driven by risk and ambiguity. Since the realized probability distributions of returns are of the normal distribution type, which is fully characterized by its first two moments, preferences can be formed as mean-uncertainty type.

Figure 4 describes the uncertain asset frontier and the CML in the mean-uncertainty space. The dotted external concave frontier describes the uncertain asset frontier when ambiguity is not present, and the solid internal concave frontier describes the uncertain asset frontier when the economy is imbued with ambiguity. The shaded area is the set of all feasible portfolios in an ambiguous economy without a risk-free asset. One can observe that the minimal uncertainty that accompanies any expected return is higher when ambiguity is present. The slope of the CML follows Equation (13), which implies a linear relationship between expected return and uncertainty. The solid straight line is the CML in an ambiguous economy, and the dotted straight line is the CML in a non-ambiguous economy. Any point on the CML to the right of m implies borrowing for the risk-free rate.

Tobin's (1958) Separation theorem asserts that any investor should hold the risk-free asset and a single optimal portfolio of risky assets, i.e., the market portfolio. Equation (13) extends Tobin's theorem from risk to uncertainty. It implies that investment decisions can be broken down into two separate phases: the first concern the choice of a unique optimal uncertain asset portfolio and second is the allocation of funds to the risk-free asset and the uncertain portfolio. Investors, in this theorem, are different only in their decision regarding the proportions allocated to the risk-free asset and the uncertain portfolio. Thus, every investor holds uncertain assets in the same proportion, as defined by the market portfolio. The nature of the market portfolio in an ambiguous economy, however, is different than Tobin's market portfolio. Tobin's market portfolio is a portfolio with the minimal variance for a given expected return, whereas in the current model the optimal portfolio has the minimal uncertainty for a given expected return, but not necessarily the minimal variance.

It is because individuals are different in their attitude toward uncertainty that the proportions of the risk-free asset and the market portfolio are different. More conservative investors, 


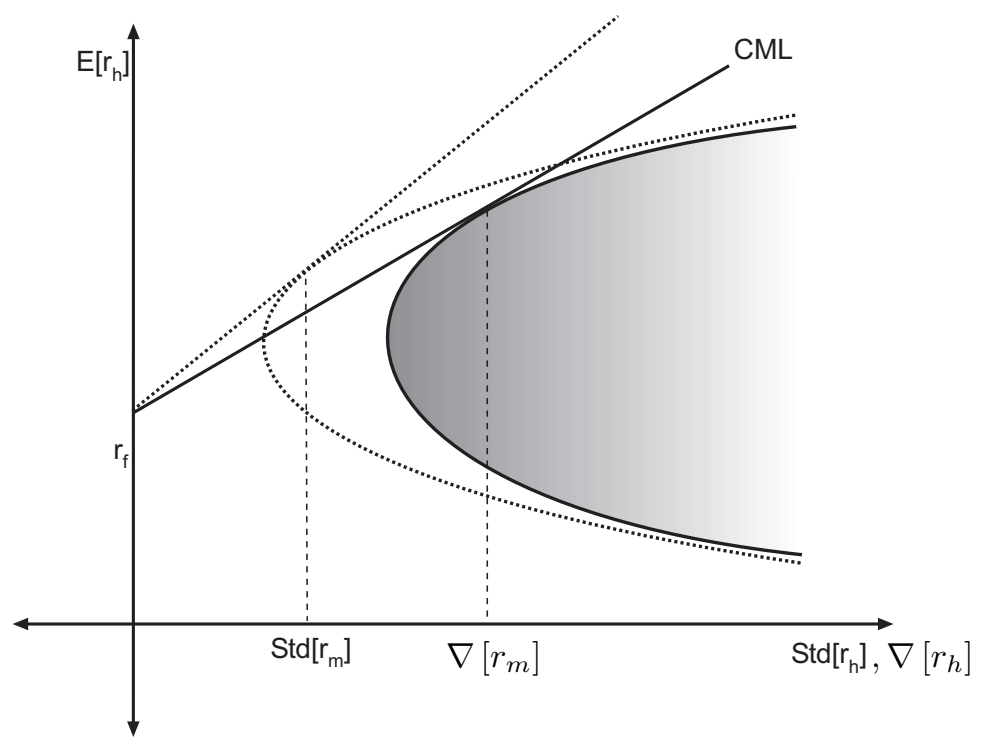

Figure 4: The uncertain asset frontier and the CML

This figure describes the uncertain asset frontier and the CML in the mean-uncertainty space. The dotted external frontier describes the risky asset frontier in a non-ambiguous economy, and the solid internal frontier describes the uncertain asset frontier in an ambiguous economy. The shaded area is the set of feasible portfolios in a non-ambiguous economy without a risk-free asset. The solid straight line is the CML in an ambiguous economy, and the dotted straight line is the CML in a non-ambiguous economy.

for example, will choose to allocate a higher fraction of their wealth to the risk-free asset. More aggressive investors may decide to borrow capital on the money market, i.e., make a negative allocation to the risk-free asset, and invest it in the market portfolio. In any case, every investment decision is made on the CML.

The CML defines the rewarding for an efficient portfolio per unit of the entire, systematic and idiosyncratic, uncertainty borne. The next section refines the systematic uncertainty associated with individual assets, and defines the premium per unit of systematic uncertainty borne.

\section{Capital asset pricing}

Based on the mean-uncertainty framework, this section generalizes the CAPM to incorporate ambiguity. It introduces a novel capital asset pricing model, called shadow capital asset pricing (SCAPM), in which the expected return on an asset corresponds to its uncertainty relative to the market and not to its own uncertainty. The conventional CAPM is a special case of the SCAPM, in which ambiguity is not present.

Definition 5.1. Systematic risk is the part of the risk that it is optimal not to diversify, and idiosyncratic risk is the part that it is optimal to diversify. Systematic ambiguity is the part 
of the ambiguity that it is optimal not to diversify, and idiosyncratic ambiguity is the part that it is optimal to diversify. Systematic uncertainty is the accumulation of systematic risk and systematic ambiguity. Idiosyncratic uncertainty is the accumulation of idiosyncratic risk and idiosyncratic ambiguity.

Systematic uncertainty is the minimal possible uncertainty for a given expected return. Systematic risk in SCAPM is not the part of risk that cannot be diversified; it is the part of risk that it is optimal not to diversify considering the tradeoff between risk and ambiguity. Systematic ambiguity is the optimal degree of ambiguity to bear conditional on its correlation with risk. When ambiguity is not present, risk minimization is optimal, so that the systematic risk is the non-diversifiable risk and the idiosyncratic risk is the diversifiable risk.

It is not always true that a higher risk, measured by the variance of return, or a higher ambiguity measured by $\mho^{2}$, both result in a higher expected return. First, it is always the case that only the systematic component of uncertainty, which aggregates systematic risk and systematic ambiguity, is associated with a premium in terms of additional expected return. An asset may have a high risk or a high ambiguity, but only a relatively small part of it is systematic and accordingly its premium is relatively low. Second, risk and ambiguity are not independent (see Izhakian (2012)). They may be negatively related such that increasing risk is accompanied by decreasing ambiguity, or vice versa. Whatever the case, uncertainty is not necessarily higher and nor is expected return.

The next theorem introduces a closed formed pricing formula which proves that only the systematic component of uncertainty is priced, i.e., compensation is provided only for the systematic risk and the systematic ambiguity.

Theorem 5.2. Let $r_{m}$ be the return on the market portfolio, $r_{f}$ the risk-free rate of return and $r_{j}$ the return on asset $j$. Assuming an investor averse to risk and ambiguity, whose reference point is $r_{f}$, if the returns on all assets are normally distributed with random parameters $\mu$ and $\sigma$, then the expected return on asset $j$ is

$$
\mathrm{E}\left[r_{j}\right]=r_{f}+\beta_{\mathcal{R}, j}\left(\mathrm{E}\left[r_{m}\right]-r_{f}\right)+\beta_{\mathcal{A}, j}\left(\mathrm{E}\left[r_{m}\right]-r_{f}\right)
$$

where

$$
\beta_{\mathcal{R}, j}=\frac{\operatorname{Cov}\left[r_{m}, r_{j}\right]}{\operatorname{Var}\left[r_{m}\right]}
$$

is the beta risk,

$$
\beta_{\mathcal{A}, j}=4 \frac{\operatorname{Cov}\left[\Phi\left(r_{f} ; \mu_{m}, \sigma_{m}\right), \phi\left(r_{f} ; \mu_{m}, \sigma_{m}\right)\left(\frac{\sigma_{m, j}}{\sigma_{m}^{2}}\left(\mu_{m}-r_{f}\right)-\left(\mu_{j}-r_{f}\right)\right)\right]}{1-\mho^{2}\left[r_{m}\right]}
$$


is the beta ambiguity,

$$
\Phi\left(r_{f} ; \mu_{m}, \sigma_{m}\right)=\int_{-\infty}^{r_{f}} \frac{1}{\sqrt{2 \pi \sigma_{m}^{2}}} e^{-\frac{\left(r-\mu_{m}\right)^{2}}{2 \sigma_{m}^{2}}} d r
$$

is the random cumulative probability of loss on the market portfolio, and

$$
\phi\left(r_{f} ; \mu_{m}, \sigma_{m}\right)=\frac{1}{\sqrt{2 \pi \sigma_{m}^{2}}} e^{-\frac{\left(r_{f}-\mu_{m}\right)^{2}}{2 \sigma_{m}^{2}}}
$$

is its probability density at the reference point.

Theorem 5.2 proves that the expected return on an asset is a function of its systematic uncertainty, formulated by $\beta_{\mathcal{R}}$ and $\beta_{\mathcal{A}}$. Beta risk, $\beta_{\mathcal{R}}$, is a function of the covariance between the asset's return and the market's return, computed using expected probabilities, i.e., the redacted first-order and second-order probabilities. The coefficient $\beta_{\mathcal{A}}$ is a function of the covariance between elements of probabilities, computed using second-order probabilities, i.e., the probability distribution of the random parameters $\mu$ and $\sigma$. Theorem 5.2 is empirically testable: $\beta_{\mathcal{R}}$ and $\beta_{\mathcal{A}}$ can be computed from the data.

The SCAPM decomposes the price of an asset, in terms of expected return, into three components: the price of time, the price of risk, and the price of ambiguity. The price of time, formed by $r_{f}$, is the pure interest rate on the risk-free asset. The price of risk, formed by

$$
\mathcal{R}=\frac{\operatorname{Cov}\left[r_{\mathbf{m}}, r_{j}\right]}{\operatorname{Var}\left[r_{\mathbf{m}}\right]}\left(\mathrm{E}\left[r_{\mathbf{m}}\right]-r_{f}\right)
$$

is an additional expected return per unit of systematic risk borne, referred to as the risk premium. The price of ambiguity, formed by

$$
\mathcal{A}=4 \frac{\operatorname{Cov}\left[\Phi\left(r_{f} ; \mu_{\mathbf{m}}, \sigma_{\mathbf{m}}\right), \phi\left(r_{f} ; \mu_{\mathbf{m}}, \sigma_{\mathbf{m}}\right)\left(\frac{\sigma_{\mathbf{m}, j}}{\sigma_{\mathbf{m}}^{2}}\left(\mu_{\mathbf{m}}-r_{f}\right)-\left(\mu_{j}-r_{f}\right)\right)\right]}{1-\mho^{2}\left[r_{\mathbf{m}}\right]}\left(\mathrm{E}\left[r_{\mathbf{m}}\right]-r_{f}\right),
$$

is a second additional expected return per unit of systematic ambiguity borne, referred to as the ambiguity premium. The uncertainty premium on the market portfolio $\mathbf{m}$, formed by $\mathrm{E}\left[r_{\mathbf{m}}\right]-r_{f}$, is the aggregate excess return rewarding for both risk and ambiguity borne by $\mathbf{m}$. The risk and the ambiguity premiums of asset $j$ are proportional to the uncertainty premium of $\mathbf{m}$, where the proportions are determined by the coefficients $\beta_{\mathcal{R}, j}$ and $\beta_{\mathcal{A}, j}$, respectively.

Assume for the moment a non-ambiguous economy, i.e., $\mu$ and $\sigma$ are constants. In this case, $\beta_{\mathcal{A}}=0$ and Theorem 5.2 collapses to the classical CAPM in which only a rewarding for systematic risk is porvided. ${ }^{21}$ If $\beta_{\mathcal{R}, j}=1$, then asset $j$ 's expected return equals the market's expected return, i.e., $\mathrm{E}\left[r_{j}\right]=\mathrm{E}\left[r_{\mathbf{m}}\right]$; if $\beta_{\mathcal{R}, j}>1$ then $\mathrm{E}\left[r_{j}\right]>\mathrm{E}\left[r_{\mathbf{m}}\right]$; and if $\beta_{\mathcal{R}, j}<1$ then $\mathrm{E}\left[r_{j}\right]<\mathrm{E}\left[r_{\mathbf{m}}\right]$, exactly as in the CAPM. If asset $j$ 's return is negatively correlated with the

\footnotetext{
${ }^{21}$ To see this, recall that the covariance between a constant and a random variable is always 0 .
} 
market's return, $\beta_{\mathcal{R}, j}<0$, then $\mathrm{E}\left[r_{j}\right]<r_{f}$, implying that investors hold this asset as an insurance against a decrease in the market return.

Theorem 5.2 proves that only the systematic component of ambiguity is accompanied by rewarding. The sign and intensity of the relation between the probability of loss on the market portfolio and the expected return on individual assets, formed be $\beta_{\mathcal{A}}$, is dominated by two elements. First, the higher the cumulative probability of loss on the market portfolio $\Phi\left(r_{f} ; \mu_{\mathbf{m}}, \sigma_{\mathbf{m}}\right)$ the greater $\beta_{\mathcal{A}}$. The reason for this is that when the probability of loss on the market portfolio increases, assets which are positively correlated with the market are required to provide higher premiums to induce investors to hold them. Second, assuming a positive expected excess return, i.e., $r_{f}-\mu_{\mathbf{m}} \leq 0$, a higher probability of loss implies a higher probability density $\phi\left(r_{f} ; \mu_{\mathbf{m}}, \sigma_{\mathbf{m}}\right)$ and therefore a higher $\beta_{\mathcal{A}}$. Higher values of the random covariance, $\sigma_{\mathbf{m}, j}$, between the return on asset $j$ and the return on the market portfolio $\mathbf{m}$ also implies a higher beta ambiguity, which in turn implies a higher expected return. Intuitively, $\beta_{\mathcal{A}}$ can be seen as measuring the correlation between the probability of loss of an asset and the probability of loss of the market portfolio.

An interesting point concerning expected return arises from Equation (16) . Writing the expression $\left(\frac{\sigma_{\mathbf{m}, j}}{\sigma_{\mathbf{m}}^{2}}\left(\mu_{\mathbf{m}}-r_{f}\right)-\left(\mu_{j}-r_{f}\right)\right)$ as $-\Delta=-\left(\left(\mu_{j}-r_{f}\right)-\frac{\sigma_{\mathbf{m}, j}}{\sigma_{\mathbf{m}}^{2}}\left(\mu_{\mathbf{m}}-r_{f}\right)\right), \Delta$ takes the meaning of an unexpected mean return, conditional on a probability distribution. Higher $\Delta \mathrm{s}$ imply a higher absolute value of $\beta_{\mathcal{A}, j}$. If $\Delta$ and the probability of loss of the market portfolio, $\Phi\left(r_{f} ; \mu_{\mathbf{m}}, \sigma_{\mathbf{m}}\right)$, are positively correlated then $\beta_{\mathcal{A}, j}$ has a negative impact on the expected return $\mathrm{E}\left[r_{j}\right]$; if the correlation is negative then $\beta_{\mathcal{A}, j}$ has a positive impact on $\mathrm{E}\left[r_{j}\right]$. The intuition of this relation is that a positive correlation between probability of loss and unexpected mean return compensates for the (second-order) states of nature that induce a high probability of loss. Therefore, the price of an asset with a positive correlation between $\Phi\left(r_{f} ; \mu_{\mathbf{m}}, \sigma_{\mathbf{m}}\right)$ and $\Delta$ is relatively high and, accordingly, its expected return is relatively low. Notice that $\Delta$ is not a shift of return (unexpected return), but a shift of the entire distribution, which shifts the expected return. It can be considered as a shock to parameter $\mu$, governing the probability distribution. Notice also that the $\Delta \mathrm{s}$ of an asset with a zero correlation with the market (a zero beta portfolio) can possibly be positive (see, for example, Merton (1973)).

The SCAPM allows for an asset $j$ to have $\beta_{\mathcal{R}, j} \neq 0$ and $\beta_{\mathcal{A}, j}=0$. This can happen, for example, when either the probability of return on the market portfolio or the probability of return on asset $j$ are perfectly known. It is also possible to observe an asset typified by $\beta_{\mathcal{R}, j}=0$ and $\beta_{\mathcal{A}, j} \neq 0$. This can happen, for example, when the covariance between $r_{j}$ and 
$r_{\mathbf{m}}$ is symmetrically volatile around zero. The following corollary defines the $\beta_{\mathcal{R}}$ and $\beta_{\mathcal{A}}$ of the market portfolio.

Corollary 5.3. The market portfolio $\boldsymbol{m}$ satisfies

$$
\beta_{\mathcal{R}, m}=1 \quad \text { and } \quad \beta_{\mathcal{A}, m}=0 .
$$

The beta risk, $\beta_{\mathcal{R}}$, and beta ambiguity, $\beta_{\mathcal{R}}$, of an asset portfolio are both linear inthe betas of the individual assets composing the portfolio. To see this, consider a portfolio $\mathbf{h}=\left(h_{1}, \ldots, h_{n}\right)$ consisting of $n$ assets. The expected return on portfolio $\mathbf{h}$ can be expressed by

$$
\mathrm{E}\left[r_{\mathbf{h}}\right]-r_{f}=\sum_{j=1}^{n} h_{j}\left(\mathrm{E}\left[r_{j}\right]-r_{f}\right)=\left(\mathrm{E}\left[r_{\mathbf{m}}\right]-r_{f}\right)\left(\sum_{j=1}^{n} h_{j} \beta_{\mathcal{R}, j}+\sum_{j=1}^{n} h_{j} \beta_{\mathcal{A}, j}\right),
$$

which implies a linear beta pricing model, even when ambiguity is involved. In other words, the beta risk and the beta ambiguity of an asset portfolio are equal to the weighted sum of the individual betas of the assets composing the portfolio.

The SCAPM, modeled by Equation (14), can be written as

$$
\mathrm{E}\left[r_{j}\right]-r_{f}=\beta_{\mathcal{K}, j}\left(\mathrm{E}\left[r_{\mathbf{m}}\right]-r_{f}\right)
$$

where $\beta_{\mathcal{K}, j}=\beta_{\mathcal{R}, j}+\beta_{\mathcal{A}, j}$ is referred to as the beta uncertainty. The beta uncertainty makes the distinction between the systematic uncertainty and the idiosyncratic uncertainty associated with an asset, as the following proposition suggests.

Proposition 5.4. The uncertainty associated with an asset $j$ can be decomposed by

$$
\nabla^{2}\left[r_{j}\right]=\beta_{\mathcal{K}, j}^{2} \frac{1-\mho^{2}\left[r_{m}\right]}{1-\mho^{2}\left[r_{j}\right]} \nabla^{2}\left[r_{m}\right]+\nabla^{2}[\epsilon]
$$

where $\mathrm{E}[\epsilon]=0$, and $\epsilon$ and $r_{m}$ are independent for every realized probability distribution. The term $\beta_{\mathcal{K}, j}^{2} \frac{1-\mho^{2}\left[r_{m}\right]}{1-\mho^{2}\left[r_{j}\right]} \nabla^{2}\left[r_{\boldsymbol{m}}\right]$ is the systematic uncertainty and the term $\nabla^{2}[\epsilon]$ is the idiosyncratic uncertainty.

In the special case when ambiguity is not involved, as in the classical CAPM, the risk associated with asset $j$ can be decomposed by

$$
\operatorname{Var}\left[r_{j}\right]=\beta_{\mathcal{R}, j}^{2} \operatorname{Var}\left[r_{\mathbf{m}}\right]+\operatorname{Var}[\epsilon]
$$

where $\mathrm{E}[\epsilon]=0$ and $\epsilon$ and $r_{\mathbf{m}}$ are independent. The non-diversifiable systematic risk takes the form $\beta_{\mathcal{R}, j}^{2} \operatorname{Var}\left[r_{\mathbf{m}}\right]$ and the diversifiable idiosyncratic risk takes the form $\operatorname{Var}[\epsilon]$. It is important to note that Equation (19) does not hold when stocks are imbued with ambiguity: the residual $\operatorname{Var}[\epsilon]=\operatorname{Var}\left[r_{j}\right]-\left(\beta_{\mathcal{R}, j}+\beta_{\mathcal{A}, j}\right)^{2} \operatorname{Var}\left[r_{\mathbf{m}}\right]$ cannot be interpreted as idiosyncratic risk since the systematic ambiguity factor $\beta_{\mathcal{A}, j}$ is involved. 


\section{The security market line}

In SCAPM the security market line (SLM) characterizes the linear relation between systematic uncertainty and expected return. Using Theorem 5.2, the SML of the conventional CAPM can be generalized from risk to uncertainty by incorporating ambiguity. Formally, when ambiguity is involved the SML is defined by

$$
\mathrm{E}\left[r_{j}\right]=r_{f}+\beta_{\mathcal{K}, j}\left(\mathrm{E}\left[r_{\mathbf{m}}\right]-r_{f}\right)
$$

The intercept, $r_{f}$, is the price of time; the slope, $\mathrm{E}\left[r_{\mathbf{m}}\right]-r_{f}$, is the uncertainty premium on the market portfolio; and the coefficient $\beta_{\mathcal{K}, j}$ models the level of systematic uncertainty associated with asset $j$. Figure 5 provides a graphical representation of the SML. The x-axis describes the magnitude of $\beta_{\mathcal{K}}$ and the y-axis describes expected return. The solid slope line describes the SML in an ambiguous economy and the dashed line describes it in a non-ambiguous economy. In an ambiguous economy the slope of the SML is steeper than in the case of a non-ambiguous economy, indicating a higher uncertainty premium on the market portfolio.

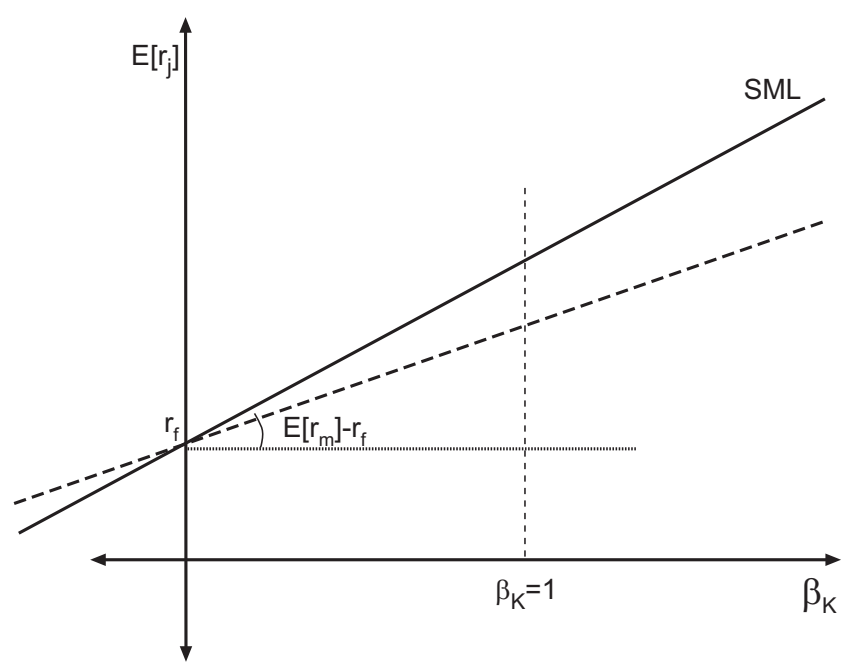

Figure 5: The security market line

This figure provides a graphical representation of the SML. The x-axis describes the magnitude of $\beta_{\mathcal{K}}$ and the y-axis describes expected return. The solid slope line describes the SML in an ambiguous economy and the dashed line describes it in a non-ambiguous economy. The SML intercepts the y-axis at the risk-free rate $r_{f}$ and its slope is equal to the uncertainty premium on the market portfolio $\mathrm{E}\left[r_{\mathbf{m}}\right]-r_{f}$.

All possible portfolios, efficient and non-efficient, lie on the SML. The reason is that the investors who minimize uncertainty for a given expected return are effectively the ones who determine the prices (and the uncertainty premiums) in the market. They are willing to pay a relatively high price for any asset, which implies a relatively low expected return, such that a 
portfolio's return is always on the SML. Another way to look at the SML is to consider it as representing the opportunity cost of various investments. Every point on the SML represents an investment in a combination of the market portfolio and the risk-free asset. Assets above the SML are considered as undervalued, since for a given amount of uncertainty they yield a relatively high return, which implies a relatively low price. Assets below the SML are considered as overvalued, since for a given amount of uncertainty they yield a relatively low return, which implies a relatively high price.

A point on the SML can be interpreted as the uncertainty premium on asset $j$ per unit of its systematic uncertainty. A natural implementation of this understanding is in measuring the performance of asset portfolios. In an economy without ambiguity the Treynor ratio measures the risk premium associated with an asset relative to its systematic risk. This ratio can now be extended to incorporate ambiguity. Equation (20) can be formed by

$$
\frac{\mathrm{E}\left[r_{j}\right]-r_{f}}{\beta_{\mathcal{K}, j}}=\mathrm{E}\left[r_{\mathbf{m}}\right]-r_{f}
$$

such that its left-hand side - the ratio of the uncertainty premium and the degree of systematic uncertainty - takes the meaning of the uncertainty premium per unit of uncertainty borne. When ambiguity is not present $\beta_{\mathcal{A}}=0$ and Equation (21) collapses to the original Treynor ratio.

A second intensively employed performance measure is the Sharpe ratio, which measures the risk premium on an asset relative to its overall, systematic and idiosyncratic, risk. Using the measure of uncertainty provided by Model 3.1, the Sharpe ratio can also be extended from risk to uncertainty. The definition of the CML in Equation (13) implies that the uncertainty premium relative to the overall, systematic and idiosyncratic, uncertainty can be measured by

$$
\frac{\mathrm{E}\left[r_{j}\right]-r_{f}}{\nabla\left[r_{j}\right]}=\frac{\mathrm{E}\left[r_{j}\right]-r_{f}}{\frac{\operatorname{Std}\left[r_{j}\right]}{\sqrt{1-\mho^{2}\left[r_{j}\right]}}} .
$$

This extended ratio characterizes the uncertainty premium on asset $j$ per unit of its entire, systematic and idiosyncratic, uncertainty.

\section{Conclusion}

This paper relaxes the main assumption of modern portfolio theory that the probabilities of returns are known. It assumes that probabilities are unknown and are themselves random. Relying on shadow theory, recently introduced by Izhakian (2011), the current paper gener- 
alizes the mean-variance space to mean-uncertainty and defines preferences in this space. It aggregates risk and ambiguity by introducing a new consolidated measure of uncertainty, which is employed to incorporate ambiguity into the classical CAPM and extend it from risk to uncertainty. A simple formalization of beta ambiguity in addition to the conventional beta risk is proved, such that systematic ambiguity is distinguished from idiosyncratic ambiguity. Capital price, in this model, is proportional to the systematic component of risk and to the systematic component of ambiguity, so that investors are not rewarded either for idiosyncratic risk or for idiosyncratic ambiguity.

The paper sheds considerable light on the relationship between the price of an asset and the various components of its overall risk and overall ambiguity. The main advantage of the model introduced in this paper is that it is empirically testable. A second advantage of the model is that it can be implemented for measuring portfolio performance by generalizing the Treynor ratio and the Sharpe ratio from risk to uncertainty. The extended ratios measure the uncertainty premium per unit of systematic uncertainty and per unit of the entire, systematic and idiosyncratic, uncertainty borne. 


\section{References}

Acharya, V. V. and L. H. Pedersen (2005) "Asset Pricing with Liquidity Risk," Journal of Financial Economics, Vol. 77, No. 2, pp. 375-410.

Bollerslev, T., R. F. Engle, and J. M. Wooldridge (1988) "A Capital Asset Pricing Model with TimeVarying Covariances," Journal of Political Economy, Vol. 96, No. 1, pp. 116-131.

Boyle, P. P., L. Garlappi, R. Uppal, and T. Wang (2011) "Keynes Meets Markowitz: The Tradeoff Between Familiarity and Diversification," Management Science, pp. 1-20.

Brenner, M. and Y. Izhakian (2011) "Asset Prices and Ambiguity: Empirical Evidance," Stern School of Business, Finance Working Paper Series, FIN-11-10.

Cao, H. H., T. Wang, and H. H. Zhang (2005) "Model Uncertainty, Limited Market Participation, and Asset Prices," Review of Financial Studies, Vol. 18, No. 4, pp. 1219-1251.

Chen, Z. and L. Epstein (2002) "Ambiguity, Risk, and Asset Returns in Continuous Time," Econometrica, Vol. 70, No. 4, pp. 1403-1443.

Cochrane, J. H. (2001) Asset Pricing, Princeton: Princeton Univ. Press.

Dow, J. and S. R. d. C. Werlang (1992) "Uncertainty Aversion, Risk Aversion, and the Optimal Choice of Portfolio," Econometrica, Vol. 60, No. 1, pp. 197-204.

Easley, D. and M. O'Hara (2009) "Ambiguity and Nonparticipation: The Role of Regulation," Review of Financial Studies, Vol. 22, No. 5, pp. 1817-1843.

Epstein, L. and M. Schneider (2008) "Ambiguity, Information Quality, and Asset Pricing," Journal of Finance, Vol. 63, No. 1, pp. 197-228.

Fama, E. F. and M. H. Miller (1972) The Theory of Finance: Holt, Rinehart and Winston New York, pp.xvi, 346 p.

Gagliardini, P., P. Porchia, and F. Trojani (2009) "Ambiguity Aversion and the Term Structure of Interest Rates," Review of Financial Studies, Vol. 22, No. 10, pp. 4157-4188.

Gilboa, I. and D. Schmeidler (1989) "Maxmin Expected Utility with Non-Unique Prior," Journal of Mathematical Economics, Vol. 18, No. 2, pp. 141-153.

Gollier, C. (2011) "Does Ambiguity Aversion Reinforce Risk Aversion? Applications to Portfolio Choices and Asset Pricing," The Review of Economic Stuies.

Guidolin, M. and F. Rinaldi (2010) "Ambiguity in Asset Pricing and Portfolio Choice: A Review of the Literature," SSRN eLibrary.

Hansen, L. P. and T. J. Sargent (2001) "Robust Control and Model Uncertainty," American Economic Review, Vol. 91, No. 2, pp. 60-66.

Izhakian, Y. (2011) "Shadow Probability Theory and Ambiguity Measurement," Preprint at SSRN:1938628.

(2012) "Shadow Probability Theory for Asset Pricing under Ambiguity," Preprint at SSRN:1937645.

Izhakian, Y. and S. Benninga (2011) "The Uncertainty Premium in an Ambiguous Economy," The Quatrly Jurnal of Finance, Vol. 1, pp. 323-354.

Ju, N. and J. Miao (2011) "Ambiguity, Learning, and Asset Returns," Econometrica.

Kahneman, D. and A. Tversky (1979) "Prospect Theory: An Analysis of Decision under Risk," Econometrica, Vol. 47, No. 2, pp. 263-91. 
Klibanoff, P., M. Marinacci, and S. Mukerji (2005) "A Smooth Model of Decision Making under Ambiguity," Econometrica, Vol. 73, No. 6, pp. 1849-1892.

- (2009) "Recursive Smooth Ambiguity Preferences," Journal of Economic Theory, Vol. 144, No. 3, pp. 930-976.

Leippold, M., F. Trojani, and P. Vanini (2008) "Learning and Asset Prices Under Ambiguous Information," Review of Financial Studies, Vol. 21, No. 6, pp. 2565-2597.

Ljungqvist, L. and T. J. Sargent (2004) Recursive Macroeconomic Theory, 2nd Edition, Vol. 1 of MIT Press Books: The MIT Press.

Maccheroni, F., M. Marinacci, and D. Ruffino (2010) "Alpha as Ambiguity: Robust Mean-Variance Portfolio Analysis."

Maccheroni, F., M. Marinacci, A. Rustichini, and M. Taboga (2009) "Portfolio Selection With Monotone Mean-Variance Preferences," Mathematical Finance, Vol. 19, No. 3, pp. 487-521.

Maenhout, P. J. (2004) "Robust Portfolio Rules and Asset Pricing," Review of Financial Studies, Vol. 17, No. 4, pp. 951-983.

Markowitz, H. (1952) "The Utility of Wealth," Journal of Political Economy, Vol. 60, p. 151. (1959) Portfolio Selection: Efficient Diversification of Investments: New York: Wiley.

Merton, R. C. (1973) "An Intertemporal Capital Asset Pricing Model," Econometrica, Vol. 41, No. 5, pp. $867-87$.

Nau, R. F. (2006) "Uncertainty Aversion with Second-Order Utilities and Probabilities," Management Science, Vol. 52, pp. 136-145.

Pflug, G. and D. Wozabal (2007) "Ambiguity in Portfolio Selection," Quantitative Finance, Vol. 7, No. 4, pp. 435-442.

Schmeidler, D. (1989) "Subjective Probability and Expected Utility without Additivity," Econometrica, Vol. 57, No. 3, pp. 571-87.

Sharpe, W. F. (1964) "Capital Asset Prices: A Theory of Market Equilibrium under Conditions of Risk," The Journal of Finance, Vol. 19, No. 3, pp. 425-442.

Taboga, M. (2005) "Portfolio Selection with Two-Stage Preferences," Finance Research Letters, Vol. 2, No. 3, pp. 152-164.

Tobin, J. (1958) "Liquidity Preference as Behavior Towards Risk," The Review of Economic Studies, Vol. 25, No. 2, pp. 65-86.

Treynor, J. L. (1961) "Market Value, Time, and Risk," Unpublished manuscript dated 8/8/61, No. 95-209.

Tversky, A. and D. Kahneman (1992) "Advances in Prospect Theory: Cumulative Representation of Uncertainty," Journal of Risk and Uncertainty, Vol. 5, No. 4, pp. 297-323.

Ui, T. (2011) "The Ambiguity Premium vs. the Risk Premium under Limited Market Participation," Review of Finance, Vol. 15, No. 2, pp. 245-275.

Uppal, R. and T. Wang (2003) "Model Misspecification and Under Diversification," Journal of Finance, Vol. 58. 


\section{A Appendix}

Proof of Proposition 3.2. To prove that the line defined by

$$
\left(\mathrm{E}\left[h r_{f}+(1-h) r_{\mathbf{e}}\right], \operatorname{Std}\left[h r_{f}+(1-h) r_{\mathbf{e}}\right], \widehat{\mho}\left[h r_{f}+(1-h) r_{\mathbf{e}}\right]\right),
$$

is linear, it has to be shown that each coordinate of this triplet is linear in $h$. The proof that the first two coordinates, the mean $\mathrm{E}\left[h r_{f}+(1-h) r_{\mathbf{e}}\right]$ and the standard deviation Std $\left[h r_{f}+(1-h) r_{\mathbf{e}}\right]$, are linear in $h$ is trivial, since $r_{f}$ is constant. To prove that the third coordinate, $\widehat{\mho}\left[h r_{f}+(1-h) r_{\mathbf{e}}\right]$, is also linear in $h$, the measure of ambiguity $\mho^{2}$ can be written explicitly

$$
\mho^{2}\left[h r_{f}+(1-h) r_{\mathbf{e}}\right]=4 \operatorname{Var}\left[\int_{-\infty}^{r_{f}} \frac{1}{\sqrt{2 \pi(1-h)^{2} \sigma_{\mathbf{e}}^{2}}} e^{-\frac{\left(x-\left((1-h) \mu_{\mathbf{e}}-h r_{f}\right)\right)^{2}}{2(1-h)^{2} \sigma_{\mathbf{e}}^{2}}} d x\right] .
$$

Changing the integration variable implies that

$$
\mho^{2}\left[h r_{f}+(1-h) r_{\mathbf{e}}\right]=4 \operatorname{Var}\left[\int_{-\infty}^{r_{f}} \frac{1}{\sqrt{2 \pi \sigma_{\mathbf{e}}^{2}}} e^{-\frac{\left(x-\mu_{\mathbf{e}}\right)^{2}}{2 \sigma_{\mathbf{e}}^{2}}} d x\right]=\mho^{2}\left[r_{\mathbf{e}}\right] .
$$

Since $r_{f}$ is constant, $\operatorname{Std}\left[h r_{f}+(1-h) r_{\mathbf{e}}\right]=(1-h) \operatorname{Std}\left[r_{\mathbf{e}}\right]$, which implies

$$
\widehat{\mho}\left[h r_{f}+(1-h) r_{\mathbf{e}}\right]=(1-h) \mho \mho^{2}\left[r_{\mathbf{e}}\right] .
$$

Proof of Theorem 4.2. Using matrix notation to write the minimization problem provides

$$
\min _{\mathbf{h}} \mathbf{h}^{T} \frac{\mathbf{C}}{1-4 \operatorname{Var}\left[\Phi\left(-\frac{\mathbf{h}^{T} \mu}{\mathbf{h}^{T} \Sigma \mathbf{h}} ; 0,1\right)\right]} \mathbf{h} \quad \text { s.t. } \quad \mathbf{E}^{T} \mathbf{h}=E ; \quad \mathbf{1}^{T} \mathbf{h}=1,
$$

where bold font designates vectors and matrixes, $\mu$ is a vector of random means, $\Sigma$ is a random variance-covariance matrix, $\mathbf{C}$ is the expected variance-covariance matrix, and $E$ is a constant. Letting $\lambda$ and $\kappa$ be the Lagrangian multiplier, the the first-order condition of the minimization problem is

$$
\begin{aligned}
2 \mathbf{h}^{T} \mathbf{C}\left(1-4 \operatorname{Var}\left[\Phi\left(-\frac{\mathbf{h}^{T} \mu}{\mathbf{h}^{T} \Sigma \mathbf{h}} ; 0,1\right)\right]\right)+2 \mathbf{h}^{T} \mathbf{C h} \operatorname{Cov}\left[\Phi\left(-\frac{\mathbf{h}^{T} \mu}{\mathbf{h}^{T} \Sigma \mathbf{h}} ; 0,1\right), \phi\left(-\frac{\mathbf{h}^{T} \mu}{\mathbf{h}^{T} \Sigma \mathbf{h}} ; 0,1\right)\left(\frac{\mu \mathbf{h}^{T} \Sigma \mathbf{h}-\mathbf{h}^{T} \mu \mathbf{h}^{T} \Sigma}{\left(\mathbf{h}^{T} \Sigma \mathbf{h}\right)^{2}}\right)\right] & \\
\left(1-4 \operatorname{Var}\left[\Phi\left(-\frac{\mathbf{h}^{T} \mu}{\mathbf{h}^{T} \Sigma \mathbf{h}} ; 0,1\right)\right]\right)^{2} & -\lambda \mathbf{E}^{T}-\kappa \mathbf{1}^{T}=0 .
\end{aligned}
$$

One can see that Equation (23) has a solution if the following two conditions hold. First, as $\mathbf{C}$ is not singular, it has an inverse matrix. Second, the degree of ambiguity is not equal to 1. That is, $1-4 \operatorname{Var}\left[\Phi\left(-\frac{\mathbf{h}^{T} \mu}{\mathbf{h}^{T} \Sigma \mathbf{h}} ; 0,1\right)\right]=1-\mho^{2}\left[r_{\mathbf{h}}\right] \neq 0$. These conditions are satisfied by the assumptions of the theorem so that the uncertain asset frontier exists. Theorem 4.3 proves that the frontier is bounded by a hyperbola shape, which implies that there exists a line stretching from $r_{f}$ and tangent to the uncertain asset frontier. 
Proof of Theorem 4.3. For simplicity this proof considers a portfolio consisting of two assets. It can then be extended to any number of assets by considering each of the two assets as a mutual fund. Let $\mathbf{h}=(h, 1-h)$ be a portfolio, with an expected return of

$$
\mathrm{E}\left[r_{\mathbf{h}}\right]=h \mathrm{E}\left[r_{1}\right]+(1-h) \mathrm{E}\left[r_{2}\right]
$$

and a variance of

$$
\operatorname{Var}\left[r_{\mathbf{h}}\right]=h^{2} \operatorname{Var}\left[r_{1}\right]+2 h(1-h) \operatorname{Cov}\left[r_{1}, r_{2}\right]+(1-h)^{2} \operatorname{Var}\left[r_{2}\right]
$$

where $r_{1}$ and $r_{2}$ stand for the return on asset 1 and 2 , respectively.

By Model 3.1 the degree of uncertainty associated with portfolio $\mathbf{h}$ is

$$
\nabla^{2}\left[r_{\mathbf{h}}\right]=\frac{h^{2} \operatorname{Var}\left[r_{1}\right]+2 h(1-h) \operatorname{Cov}\left[r_{1}, r_{2}\right]+(1-h)^{2} \operatorname{Var}\left[r_{2}\right]}{1-4 \operatorname{Var}\left[\Phi\left(\frac{r_{f}-h \mu_{1}-(1-h) \mu_{2}}{\sqrt{h^{2} \sigma_{1}^{2}+2 h(1-h) \sigma_{1,2}+(1-h)^{2} \sigma_{2}^{2}}} ; 0,1\right)\right]} .
$$

Substituting for $h=0$ provides the degree of uncertainty associated with asset 1 :

$$
\nabla^{2}\left[r_{1}\right]=\frac{\operatorname{Var}\left[r_{1}\right]}{1-4 \operatorname{Var}\left[\Phi\left(\frac{r_{f}-\mu_{1}}{\sigma_{1}} ; 0,1\right)\right]} .
$$

Substituting for $h=1$ provides the degree of uncertainty associated with asset 2 :

$$
\nabla^{2}\left[r_{2}\right]=\frac{\operatorname{Var}\left[r_{2}\right]}{1-4 \operatorname{Var}\left[\Phi\left(\frac{r_{f}-\mu_{2}}{\sigma_{2}} ; 0,1\right)\right]}
$$

Since $\mho^{2}[r]=4 \operatorname{Var}\left[\Phi\left(\frac{r_{f}-\mu}{\sigma} ; 0,1\right)\right] \in[0,1]$, the degree of uncertainty satisfies $\nabla^{2}\left[r_{1}\right] \geq \operatorname{Var}\left[r_{1}\right]$ and $\nabla^{2}\left[r_{2}\right] \geq \operatorname{Var}\left[r_{2}\right]$.

Since the parameters $\mu_{1}$ and $\mu_{2}$ are random, the proportions of the two assets in the portfolio are selected according to their expected return. By Equation (24), the proportion of asset 1 in the portfolio can be writing as $h=\frac{\mathrm{E}\left[r_{1}\right]-\mathrm{E}\left[r_{2}\right]}{\mathrm{E}\left[r_{1}\right]-\mathrm{E}\left[r_{2}\right]}$. Substituting this proportion into Equation (26) produces

$$
\nabla^{2}\left[r_{\mathbf{h}}\right]=\frac{\left(\frac{\mathrm{E}\left[r_{\mathbf{h}}\right]-\mathrm{E}\left[r_{2}\right]}{\mathrm{E}\left[r_{1}\right]-\mathrm{E}\left[r_{2}\right]}\right)^{2} \operatorname{Var}\left[r_{1}\right]+2 \frac{\mathrm{E}\left[r_{\mathbf{h}}\right]-\mathrm{E}\left[r_{2}\right]}{\mathrm{E}\left[r_{1}\right]-\mathrm{E}\left[r_{2}\right]}\left(1-\frac{\mathrm{E}\left[r_{\mathbf{h}}\right]-\mathrm{E}\left[r_{2}\right]}{\mathrm{E}\left[r_{1}\right]-\mathrm{E}\left[r_{2}\right]}\right) \operatorname{Cov}\left[r_{1}, r_{2}\right]+\left(1-\frac{\mathrm{E}\left[r_{\mathbf{h}}\right]-\mathrm{E}\left[r_{2}\right]}{\mathrm{E}\left[r_{1}\right]-\mathrm{E}\left[r_{2}\right]}\right)^{2} \operatorname{Var}\left[r_{2}\right]}{1-\operatorname{Var}\left[\Phi\left(\frac{r_{f}-\frac{\mathrm{E}\left[r_{\mathbf{h}}\right]-\mathrm{E}\left[r_{2}\right]}{\mathrm{E}\left[r_{1}\right]-\mathrm{E}\left[r_{2}\right]} \mu_{1}-\left(1-\frac{\mathrm{E}\left[r_{\mathbf{h}}\right]-\mathrm{E}\left[r_{2}\right]}{\mathrm{E}\left[r_{1}\right]-\mathrm{E}\left[r_{2}\right]}\right) \mu_{2}}{\sqrt{\left(\frac{\mathrm{E}\left[r_{\mathbf{h}}\right]-\mathrm{E}\left[r_{2}\right]}{\mathrm{E}\left[r_{1}\right]-\mathrm{E}\left[r_{2}\right]}\right)^{2} \sigma_{1}^{2}+2 \frac{\mathrm{E}\left[r_{\mathbf{h}}\right]-\mathrm{E}\left[r_{2}\right]}{\mathrm{E}\left[r_{1}\right]-\mathrm{E}\left[r_{2}\right]}\left(1-\frac{\mathrm{E}\left[r_{\mathbf{h}}\right]-\mathrm{E}\left[r_{2}\right]}{\mathrm{E}\left[r_{1}\right]-\mathrm{E}\left[r_{2}\right]}\right) \sigma_{1,2}+\left(1-\frac{\mathrm{E}\left[r_{\mathbf{h}}\right]-\mathrm{E}\left[r_{2}\right]}{\mathrm{E}\left[r_{1}\right]-\mathrm{E}\left[r_{2}\right]}\right)^{2} \sigma_{2}^{2}}} ; 0,1\right)\right]},
$$

which implies

$$
\nabla^{2}\left[r_{\mathbf{h}}\right]=\left(\frac{1}{\mathrm{E}\left[r_{1}\right]-\mathrm{E}\left[r_{2}\right]}\right)^{2} \frac{\left(\mathrm{E}\left[r_{\mathbf{h}}\right]-\mathrm{E}\left[r_{2}\right]\right)^{2} \operatorname{Var}\left[r_{1}\right]-2\left(\mathrm{E}\left[r_{\mathbf{h}}\right]-\mathrm{E}\left[r_{2}\right]\right)\left(\mathrm{E}\left[r_{\mathbf{h}}\right]-\mathrm{E}\left[r_{1}\right]\right) \operatorname{Cov}\left[r_{1}, r_{2}\right]+\left(\mathrm{E}\left[r_{\mathbf{h}}\right]-\mathrm{E}\left[r_{1}\right]\right)^{2} \operatorname{Var}\left[r_{2}\right]}{1-\operatorname{Var}\left[\Phi\left(\frac{r_{f}\left(\mathrm{E}\left[r_{1}\right]-\mathrm{E}\left[r_{2}\right]\right)-\left(\mathrm{E}\left[r_{\mathbf{h}}\right]-\mathrm{E}\left[r_{2}\right]\right) \mu_{1}+\left(\mathrm{E}\left[r_{\mathbf{h}}\right]-\mathrm{E}\left[r_{1}\right]\right) \mu_{2}}{\sqrt{\left(\mathrm{E}\left[r_{\mathbf{h}}\right]-\mathrm{E}\left[r_{2}\right]\right)^{2} \sigma_{1}^{2}-2\left(\mathrm{E}\left[r_{\mathbf{h}}\right]-\mathrm{E}\left[r_{2}\right]\right)\left(\mathrm{E}\left[r_{\mathbf{h}}\right]-\mathrm{E}\left[r_{1}\right]\right) \sigma_{1,2}+\left(\mathrm{E}\left[r_{\mathbf{h}}\right]-\mathrm{E}\left[r_{1}\right]\right)^{2} \sigma_{2}^{2}}} ; 0,1\right)\right]}
$$

The numerator of Equation (27) forms a parabola in the mean-uncertainty, $\mathrm{E}[r]-\nabla^{2}[r]$, space, such that its square root is a hyperbola in the $\mathrm{E}[r]-\nabla[r]$ space. The denominator ranges between 0 and 1 , implying that $\nabla^{2}\left[r_{\mathbf{h}}\right] \geq \operatorname{Var}\left[r_{\mathbf{h}}\right]$ for any $h$. 
Proof of Theorem 5.2. The random probability of loss, $\mathrm{P}\left(L_{\mathbf{m}}\right)=\mathrm{P}\left(r_{\mathbf{m}} \leq r_{f}\right)$, on the market portfolio, $\mathbf{m}$, is defined by

$$
\mathrm{P}\left(L_{\mathbf{m}}\right)=\int_{-\infty}^{r_{f}} \frac{1}{\sqrt{2 \pi \sigma_{\mathbf{m}}^{2}}} e^{-\frac{\left(r-\mu_{\mathbf{m}}\right)^{2}}{2 \sigma_{\mathbf{m}}^{2}}} d r .
$$

First, we assemble a portfolio, denoted a, consisting of a proportion $-h$ of the risk-free asset, and the remainder, $1+h$, is allocated to the uncertain market portfolio, where $h>0$. The expected return on portfolio $\mathbf{a}$ is

$$
\mathrm{E}\left[r_{\mathbf{a}}\right]=(1+h) \mathrm{E}\left[r_{\mathbf{m}}\right]-h r_{f}
$$

The variance of portfolio a's return is

$$
\operatorname{Var}\left[r_{\mathbf{a}}\right]=(1+h)^{2} \operatorname{Var}\left[r_{\mathbf{m}}\right]
$$

and its degree of ambiguity is

$$
\mho^{2}\left[r_{\mathbf{a}}\right]=4 \operatorname{Var}\left[\int_{-\infty}^{r_{f}} \frac{1}{\sqrt{2 \pi(1+h)^{2} \sigma_{\mathbf{m}}^{2}}} e^{-\frac{\left(r-\left((1+h) \mu_{\mathbf{m}}-h r_{f}\right)\right)^{2}}{2(1+h)^{2} \sigma_{\mathbf{m}}^{2}}} d r\right] .
$$

We also assemble a second portfolio, denoted $\mathbf{b}$, consisting of a portion 1 of $\mathbf{m}$ and a portion $0<h$ of some asset $j$, which is financed by a portion $h$ of the risk-free asset. Asset $j$ is assumed to be non-efficient; in the mean-uncertainty space it lies in the set of feasible portfolios, but not on the efficient frontier. The expected return on portfolio $\mathbf{b}$ is

$$
\mathrm{E}\left[r_{\mathbf{b}}\right]=\mathrm{E}\left[r_{\mathbf{m}}\right]+h \mathrm{E}\left[r_{j}\right]-h r_{f}
$$

and its variance is

$$
\operatorname{Var}\left[r_{\mathbf{b}}\right]=\operatorname{Var}\left[r_{\mathbf{m}}\right]+h^{2} \operatorname{Var}\left[r_{j}\right]+2 h \operatorname{Cov}\left[r_{\mathbf{m}}, r_{j}\right]
$$

The ambiguity associated with portfolio $\mathbf{b}$ is formed by

$$
\begin{aligned}
\mho^{2}\left[r_{\mathbf{b}}\right] & =4 \operatorname{Var}\left[\int_{-\infty}^{r_{f}} \frac{1}{\sqrt{2 \pi\left(\sigma_{\mathbf{m}}^{2}+h^{2} \sigma_{j}^{2}+2 h \sigma_{\mathbf{m}, j}\right)}} e^{-\frac{\left(r-\left(\mu_{\mathbf{m}}+h \mu_{j}-h r_{f}\right)\right)^{2}}{2\left(\sigma_{\mathbf{m}}^{2}+h^{2} \sigma_{j}^{2}+2 h \sigma_{\mathbf{m}, j}\right)}} d r\right] \\
& =4 \operatorname{Var}\left[\int_{-\infty}^{\frac{(1+h) r_{f}-\mu_{\mathbf{m}}-h \mu_{j}}{\sqrt{\sigma_{\mathbf{m}}^{2}+h^{2} \sigma_{j}^{2}+2 h \sigma_{\mathbf{m}, j}}}} \frac{1}{\sqrt{2 \pi}} e^{-\frac{(r)^{2}}{2}} d r\right],
\end{aligned}
$$

where the second equality is obtained by changing the integration variable.

As $h \rightarrow 0$, the curve drawn by portfolio a tangents the CML at the point $\left(\mathrm{E}\left[r_{\mathbf{m}}\right], \nabla\left[r_{\mathbf{m}}\right]\right)$. Thus

$$
\left.\frac{\frac{d \mathrm{E}\left[r_{\mathbf{a}}\right]}{d h}}{\frac{d \sqrt{\frac{\operatorname{Var}\left[r_{\mathbf{a}}\right]}{1-\mho^{2}\left[r_{\mathbf{a}}\right]}}}{d h}}\right|_{h=0}=\frac{\mathrm{E}\left[r_{\mathbf{m}}\right]-r_{f}}{\sqrt{\frac{\operatorname{Var}\left[r_{\mathbf{m}}\right]}{1-\mho^{2}\left[r_{\mathbf{m}}\right]}}}
$$


The derivative of the numerator in the left-hand side of Equation (30) with respect to $h$ satisfies

$$
\frac{d \mathrm{E}\left[r_{\mathbf{a}}\right]}{d h}=\mathrm{E}\left[r_{\mathbf{m}}\right]-r_{f}
$$

Since changing the integration variable of Equation (28) implies that

$$
\int_{-\infty}^{r_{f}} \frac{1}{\sqrt{2 \pi(1+h)^{2} \sigma_{\mathbf{m}}^{2}}} e^{-\frac{\left(r-\left((1+h) \mu_{\mathbf{m}}-h r_{f}\right)\right)^{2}}{2(1+h)^{2} \sigma_{\mathbf{m}}^{2}}} d r=\int_{-\infty}^{r_{f}} \frac{1}{\sqrt{2 \pi \sigma_{\mathbf{m}}^{2}}} e^{-\frac{\left(r-\mu_{\mathbf{m}}\right)^{2}}{2 \sigma_{\mathbf{m}}^{2}}} d r
$$

(see Proposition 3.2), the derivative of the denominator in the left-hand side of Equation (30) with respect to $h$ satisfies

$$
\left.\frac{d \sqrt{\frac{\operatorname{Var}\left[r_{\mathbf{a}}\right]}{1-\mho^{2}\left[r_{\mathbf{a}}\right]}}}{d h}\right|_{h=0}=\frac{\frac{\operatorname{Var}\left[r_{\mathbf{m}}\right]}{1-\mho^{2}\left[r_{\mathbf{m}}\right]}}{\sqrt{\frac{\operatorname{Var}\left[r_{\mathbf{m}}\right]}{1-\mho^{2}\left[r_{\mathbf{m}}\right]}}}
$$

Together, Equations (31) and (32) imply that

$$
\left.\frac{\frac{d \mathrm{E}\left[r_{\mathbf{a}}\right]}{d h}}{\frac{d \sqrt{\frac{\operatorname{Var}\left[r_{\mathbf{a}}\right]}{1+\mho^{2}\left[r_{\mathbf{a}}\right]}}}{d h}}\right|_{h=0}=\frac{\mathrm{E}\left[r_{\mathbf{m}}\right]-r_{f}}{\sqrt{\frac{\operatorname{Var}\left[r_{\mathbf{m}}\right]}{1-\mho^{2}\left[r_{\mathbf{m}}\right]}}} .
$$

As $h \rightarrow 0$, the curve drawn by portfolio $\mathbf{b}$ also tangents the CML at the point $\left(\mathrm{E}\left[r_{\mathbf{m}}\right], \nabla\left[r_{\mathbf{m}}\right]\right)$. Thus

$$
\left.\frac{\frac{d \mathrm{E}\left[r_{\mathbf{b}}\right]}{d h}}{\frac{d \sqrt{\frac{\operatorname{Var}\left[r_{\mathbf{b}}\right]}{1+\mho^{2}\left[r_{\mathbf{b}}\right]}}}{d h}}\right|_{h=0}=\frac{\mathrm{E}\left[r_{\mathbf{m}}\right]-r_{f}}{\sqrt{\frac{\operatorname{Var}\left[r_{\mathbf{m}}\right]}{1-\mho^{2}\left[r_{\mathbf{m}}\right]}}}
$$

The derivative of the numerator in the left-hand side of Equation (34) with respect to $h$ satisfies

$$
\frac{d \mathrm{E}\left[r_{\mathbf{b}}\right]}{d h}=\mathrm{E}\left[r_{j}\right]-r_{f}
$$

The derivative of the denominator in the left-hand side of Equation (34) with respect to $h$ satisfies $^{22}$

$\left.\frac{d \sqrt{\frac{\operatorname{Var}\left[r_{\mathbf{b}}\right]}{1-\mho^{2}\left[r_{\mathbf{b}}\right]}}}{d h}\right|_{h=0}=\left(\begin{array}{l}\frac{\operatorname{Cov}\left[r_{\mathbf{m}}, r_{j}\right]}{1-\mho^{2}\left[r_{\mathbf{m}}\right]}+4 \frac{\operatorname{Var}\left[r_{\mathbf{m}}\right]}{\left(1-\mho^{2}\left[r_{\mathbf{m}}\right]\right)^{2}} \times \\ \sum_{i=1}^{m} \chi_{i}\left(\mathrm{P}\left(L_{\mathbf{b}}\right)-\sum_{i=1}^{m} \mathrm{P}\left(L_{\mathbf{b}}\right)\right)\left(\mathrm{P}^{\prime}\left(L_{\mathbf{b}}\right)-\sum_{i=1}^{m} \mathrm{P}^{\prime}\left(L_{\mathbf{b}}\right)\right)\end{array}\right) \frac{1}{\sqrt{\frac{\operatorname{Var}\left[r_{\mathbf{m}}\right]}{1-\mho^{2}\left[r_{\mathbf{m}}\right]}}}$,

where $\chi$ is the second-order probability,

$$
\left.\mathrm{P}\left(L_{\mathbf{b}}\right)\right|_{h=0}=\int_{-\infty}^{\frac{r_{f}-\mu_{\mathbf{m}}}{\sigma_{\mathbf{m}}}} \frac{1}{\sqrt{2 \pi}} e^{-\frac{r^{2}}{2}} d r=\mathrm{P}\left(L_{\mathbf{m}}\right)
$$

and

$$
\begin{aligned}
\left.\mathrm{P}^{\prime}\left(L_{\mathbf{b}}\right)\right|_{h=0} & =\frac{1}{\sqrt{2 \pi}} e^{-\frac{\left(r_{f}-\mu_{\mathbf{m}}\right)^{2}}{2 \sigma_{\mathbf{m}}^{2}}}\left(\frac{r_{f}-\mu_{j}}{\sigma_{\mathbf{m}}}+\frac{\sigma_{\mathbf{m}, j}}{\sigma_{\mathbf{m}}^{3}}\left(\mu_{\mathbf{m}}-r_{f}\right)\right) \\
& =\phi\left(r_{f} ; \mu_{\mathbf{m}}, \sigma_{\mathbf{m}}\right)\left(\frac{\sigma_{\mathbf{m}, j}}{\sigma_{\mathbf{m}}^{2}}\left(\mu_{\mathbf{m}}-r_{f}\right)-\left(\mu_{j}-r_{f}\right)\right) .
\end{aligned}
$$

\footnotetext{
${ }^{22}$ To save on notations the descriptor $i$, designating random probability measure $\mathrm{P}_{i}$, is omitted.
} 
Equation (36) then becomes

$\left.\frac{d \sqrt{\frac{\operatorname{Var}\left[r_{\mathbf{b}}\right]}{1-\mho^{2}\left[r_{\mathbf{b}}\right]}}}{d h}\right|_{h=0}=\left(\begin{array}{l}\frac{\operatorname{Cov}\left[r_{\mathbf{m}}, r_{j}\right]}{1-\mho^{2}\left[r_{\mathbf{m}}\right]}+4 \frac{\operatorname{Var}\left[r_{\mathbf{m}}\right]}{\left(1-\mho^{2}\left[r_{\mathbf{m}}\right]\right)^{2}} \times \\ \operatorname{Cov}\left[\Phi\left(r_{f} ; \mu_{\mathbf{m}}, \sigma_{\mathbf{m}}\right), \phi\left(r_{f} ; \mu_{\mathbf{m}}, \sigma_{\mathbf{m}}\right)\left(\frac{\sigma_{\mathbf{m}, j}}{\sigma_{\mathbf{m}}^{2}}\left(\mu_{\mathbf{m}}-r_{f}\right)-\left(\mu_{j}-r_{f}\right)\right)\right]\end{array}\right) \frac{1}{\sqrt{\frac{\operatorname{Var}\left[r_{\mathbf{m}}\right]}{1-\mho^{2}\left[r_{\mathbf{m}}\right]}}}$,

which implies

$$
\left.\frac{\frac{d \mathrm{E}\left[r_{\mathbf{b}}\right]}{d h}}{\frac{d \sqrt{\frac{\operatorname{Var}\left[r_{\mathbf{b}}\right]}{1+\mho^{2}\left[r_{\mathbf{b}}\right]}}}{d h}}\right|_{h=0}=\frac{\mathrm{E}\left[r_{j}\right]-r_{f}}{\frac{\frac{\operatorname{Cov}\left[r_{\mathbf{m}}, r_{j}\right]}{1-\mho^{2}\left[r_{\mathbf{m}}\right]}+4 \frac{\operatorname{Var}\left[r_{\mathbf{m}}\right]}{\left(1-\mho^{2}\left[r_{\mathbf{m}}\right]\right)^{2}} \operatorname{Cov}\left[\Phi\left(r_{f} ; \mu_{\mathbf{m}}, \sigma_{\mathbf{m}}\right), \phi\left(r_{f} ; \mu_{\mathbf{m}}, \sigma_{\mathbf{m}}\right)\left(\frac{\sigma_{\mathbf{m}}, j}{\sigma_{\mathbf{m}}^{2}}\left(\mu_{\mathbf{m}}-r_{f}\right)-\left(\mu_{j}-r_{f}\right)\right)\right]}{\sqrt{\frac{\operatorname{Var}\left[r_{\mathbf{m}}\right]}{1-\mho^{2}\left[r_{\mathbf{m}}\right]}}}} .
$$

Equating Equations (33) and (37) yields

$$
\frac{\mathrm{E}\left[r_{j}\right]-r_{f}}{\frac{\operatorname{Cov}\left[r_{\mathbf{m}}, r_{j}\right]}{1-\mho^{2}\left[r_{\mathbf{m}}\right]}+4 \frac{\operatorname{Var}\left[r_{\mathbf{m}}\right]}{\left(1-\mho^{2}\left[r_{\mathbf{m}}\right]\right)^{2}} \operatorname{Cov}\left[\Phi\left(r_{f} ; \mu_{\mathbf{m}}, \sigma_{\mathbf{m}}\right), \phi\left(r_{f} ; \mu_{\mathbf{m}}, \sigma_{\mathbf{m}}\right)\left(\frac{\sigma_{\mathbf{m}, j}}{\sigma_{\mathbf{m}}^{2}}\left(\mu_{\mathbf{m}}-r_{f}\right)-\left(\mu_{j}-r_{f}\right)\right)\right]}=\frac{\mathrm{E}\left[r_{\mathbf{m}}\right]-r_{f}}{\frac{\operatorname{Var}\left[r_{\mathbf{m}}\right]}{1-\mho^{2}\left[r_{\mathbf{m}}\right]}} .
$$

Arranging terms provides

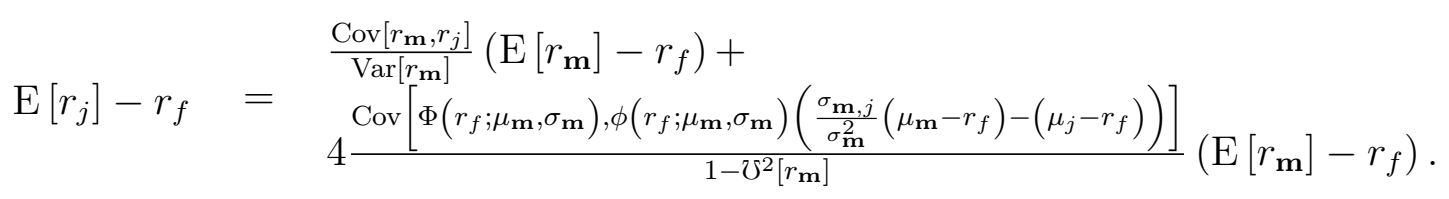

Proof of Corollary 5.3. As regards $\beta_{\mathcal{R}, \mathbf{m}}=1$, it is trivial. As regards $\beta_{\mathcal{A}, \mathbf{m}}$, from Equation (16) one can see that substituting $\mu_{j}=\mu_{\mathbf{m}}$ and $\sigma_{\mathbf{m}, j}=\sigma_{\mathbf{m}, \mathbf{m}}$ into $\left(\left(\mu_{j}-r_{f}\right)-\frac{\sigma_{\mathbf{m}, j}}{\sigma_{\mathbf{m}}^{2}}\left(\mu_{\mathbf{m}}-r_{f}\right)\right)$ implies $\beta_{\mathcal{A}, \mathbf{m}}=0$.

Proof of Theorem 5.4. By Theorem 5.2, the return on asset $j$ can be written as

$$
r_{j}-r_{f}=\beta_{\mathcal{K}, j}\left(r_{\mathbf{m}}-r_{f}\right)+\hat{\epsilon}
$$

where $\mathrm{E}[\hat{\epsilon}]=\mathrm{E}\left[r_{\mathbf{m}} \hat{\epsilon}\right]=0$, which implies that $\mathrm{E}\left[r_{j}-r_{f}\right]=\beta_{\mathcal{K}, j} \mathrm{E}\left[r_{\mathbf{m}}-r_{f}\right]$. Taking the variance, using expected probabilities, of both sides of Equation (38) yields

$$
\operatorname{Var}\left[r_{j}\right]=\beta_{\mathcal{K}, j}^{2} \operatorname{Var}\left[r_{\mathbf{m}}\right]+\operatorname{Var}[\hat{\epsilon}]
$$

Normalizing by $1-\mho^{2}\left[r_{j}\right]$ provides

$$
\frac{\operatorname{Var}\left[r_{j}\right]}{1-\mho^{2}\left[r_{j}\right]}=\beta_{\mathcal{K}, j}^{2}\left(\frac{1-\mho^{2}\left[r_{\mathbf{m}}\right]}{1-\mho^{2}\left[r_{j}\right]}\right)\left(\frac{\operatorname{Var}\left[r_{\mathbf{m}}\right]}{1-\mho^{2}\left[r_{\mathbf{m}}\right]}\right)+\frac{\operatorname{Var}[\hat{\epsilon}]}{1-\mho^{2}\left[r_{j}\right]} .
$$

Normalizing $\hat{\epsilon}$ by $\epsilon=\hat{\epsilon} \sqrt{\frac{1-\mho^{2}[\epsilon]}{1-\mho^{2}\left[r_{j}\right]}}$ yields

$$
\nabla^{2}\left[r_{j}\right]=\beta_{\mathcal{K}, j}^{2} \frac{1-\mho^{2}\left[r_{\mathbf{m}}\right]}{1-\mho^{2}\left[r_{j}\right]} \nabla^{2}\left[r_{\mathbf{m}}\right]+\nabla^{2}[\epsilon]
$$

